\title{
Unraveling the Mott-Peierls intrigue in vanadium dioxide
}

\author{
F. Grandi $\odot,{ }^{1,2}$ A. Amaricci, ${ }^{1,3}$ and M. Fabrizio ${ }^{1}$ \\ ${ }^{1}$ Scuola Internazionale Superiore di Studi Avanzati (SISSA), Via Bonomea 265, I-34136 Trieste, Italy \\ ${ }^{2}$ Department of Physics, University of Erlangen-Nürnberg, 91058 Erlangen, Germany \\ ${ }^{3}$ CNR-IOM DEMOCRITOS, Istituto Officina dei Materiali, Consiglio Nazionale delle Ricerche, Via Bonomea 265, I-34136 Trieste, Italy
}

(Received 25 June 2019; revised manuscript received 14 November 2019; accepted 19 February 2020; published 11 March 2020)

\begin{abstract}
Vanadium dioxide is one of the most studied strongly correlated materials. Nonetheless, the intertwining between electronic correlation and lattice effects has precluded a comprehensive description of the rutile metal to monoclinic insulator transition, in turn triggering a longstanding "the chicken or the egg" debate about which comes first, the Mott localization or the Peierls distortion. Here, we suggest that this problem is in fact ill posed: The electronic correlations and the lattice vibrations conspire to stabilize the monoclinic insulator, and so they must be both considered to not miss relevant pieces of the $\mathrm{VO}_{2}$ physics. Specifically, we design a minimal model for $\mathrm{VO}_{2}$ that includes all the important physical ingredients: the electronic correlations, the multiorbital character, and the two components of the antiferrodistortive mode that condense in the monoclinic insulator. We solve this model by dynamical mean-field theory within the adiabatic Born-Oppenheimer approximation. Consistently with the first-order character of the metal-insulator transition, the Born-Oppenheimer potential has a rich landscape, with minima corresponding to the undistorted phase and to the four equivalent distorted ones, and which translates into an equally rich thermodynamics that we uncover by the Monte Carlo method. Remarkably, we find that a distorted metal phase intrudes between the low-temperature distorted insulator and high-temperature undistorted metal, which sheds new light on the debated experimental evidence of a monoclinic metallic phase.
\end{abstract}

DOI: 10.1103/PhysRevResearch.2.013298

\section{INTRODUCTION}

Vanadium dioxide $\left(\mathrm{VO}_{2}\right)$ is a transition-metal compound with tremendous potential for technological applications, essentially because of its nearly room-temperature metal-toinsulator transition [1-10]. Over the years, $\mathrm{VO}_{2}$ has been subject to an intense investigation, which dates back to the first decades of the past century [11-20], but that is ongoing [21-23] and, to some extent, debated [24-30]. At the critical temperature $T_{c} \sim 340 \mathrm{~K}$ and ambient pressure, $\mathrm{VO}_{2}$ undergoes a first-order transition from a metal $\left(T>T_{c}\right)$ to an insulator $\left(T<T_{c}\right)$ [31,32], both phases being paramagnetic [33-35]. In concomitance with the metal-insulator transition, a structural distortion occurs from a high-temperature rutile (R) structure to a low temperature monoclinic (M1) one.

The crystal structure of rutile $\mathrm{VO}_{2}$ is formed by equally spaced vanadium atoms sitting at the center of edge-sharing oxygen octahedra that form linear chains along the $\mathrm{R} c$ axis, which we shall denote as $c_{R}$; see Fig. 1 . The tetragonal crystal field splits the $3 d$ manifold into two higher $e_{g}$ and three lower $t_{2 g}$ levels. In the oxidation state $\mathrm{V}^{4+}$, the single valence electron of vanadium can, therefore, occupy any of the three

Published by the American Physical Society under the terms of the Creative Commons Attribution 4.0 International license. Further distribution of this work must maintain attribution to the author(s) and the published article's title, journal citation, and DOI. $t_{2 g}$ orbitals, which are in turn distinguished into a singlet $a_{1 g}$ (or $d_{\|}$) and a doublet $e_{g}^{\pi}$ (or $d_{\pi^{*}}$ ), having, respectively, bonding and nonbonding character along the $c_{R}$ axis. The M1 phase is instead characterized by an antiferroelectric displacement of each vanadium away from the center of the octahedra, see Fig. 1, so that the above-mentioned chains, from being straight in the $\mathrm{R}$ phase, turn zigzag and dimerize [36,37].

A simple portrait of the transition in $\mathrm{VO}_{2}$ was proposed in 1971 by Goodenough [38]. According to his proposal, the basal-plane component of the antiferroelectric distortion raises the energy of $e_{g}^{\pi}$ with respect to the $a_{1 g}$ [39]. In addition, the $c_{R}$ component of the distortion, which drives the chain dimerization, opens a hybridization gap between bonding and antibonding combinations of the $a_{1 g}$. For a sufficiently large crystal field splitting and hybridization gap, the bonding combination of the $a_{1 g}$ fills completely, while the antibonding as well as the $e_{g}^{\pi}$ get empty, and hence the insulating behavior. The Goodenough's mechanism for the metal-insulator transition in $\mathrm{VO}_{2}$ thus relies on a single-particle description: the Peierls instability of the quasi-one-dimensional $a_{1 g}$ band that becomes half-filled after the growth of the crystal field drained the $e_{g}^{\pi}$ orbital.

However, Pouget et al. [40] and later Zylbersztejn and Mott [41] soon after argued that the role of electronic correlation cannot be neglected as in the Goodenough's scenario. Indeed, a tiny $\approx 0.2 \%$ substitution of $\mathrm{V}$ with $\mathrm{Cr}$ changes the lowtemperature insulator from the M1 crystal structure to a new monoclinic phase, named M2, where dimerized and zigzag chains alternate $[34,42]$. The M2 phase can be also stabilized 


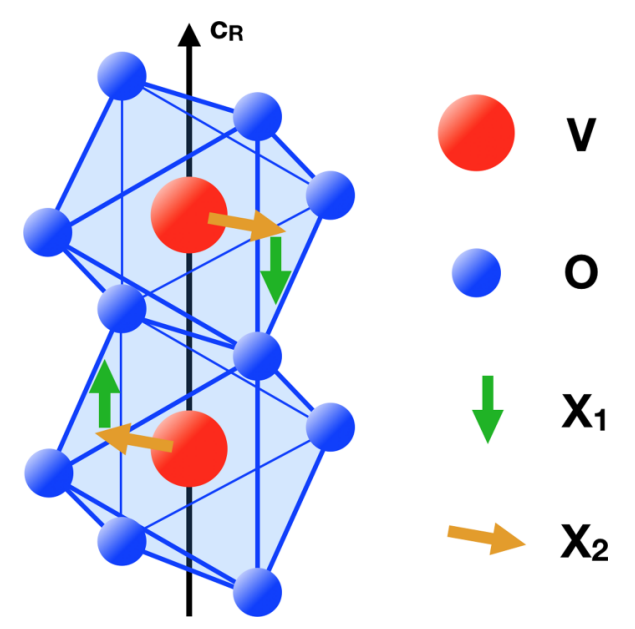

FIG. 1. The rutile crystal structure, where the large (small) spheres represent vanadium (oxygen) atoms. A cartoon of the $X_{1}$ and $X_{2}$ lattice distortions is also depicted, where the $X_{1}$ component acts as a dimerization along the $c_{R}$ axis and the $X_{2}$ component acts as a tilting in the perpendicular plane. The monoclinic M1 phase is actually characterized by finite displacements both of $X_{1}$ and $X_{2}$.

under hydrostatic pressure or uniaxial stress $[31,32,40,43-$ 45]. In addition, a triclinic ( $\mathrm{T}$ ) phase with intermediate structural properties [42] was shown to intrude between M1 and M2. The zigzag undimerized chains in M2 are still insulating and display magnetic properties akin those of a spin- $1 / 2$ antiferromagnetic Heisenberg chain $[40,42,46]$. This likeness can be rationalized only invoking sizable electronic correlations. Given the low concentration of substitutional chromium or the small value of uniaxial stress required to stabilize M2, it is reasonable to conclude that M1 must be as correlated as M2 [47-50].

We believe that, even though electronic correlations are likely necessary, they are nonetheless not sufficient to explain the phase diagram of $\mathrm{VO}_{2}$. It is known that a strong enough repulsion may drive a Mott transition in a three-band Hubbard model at the density of one electron per site [51]. Therefore, it is quite possible that the insulating phase of $\mathrm{VO}_{2}$ is driven by correlations alone and that the structural distortion below $T_{c}$ is just the best way the Mott insulator can freeze the residual spin and orbital degrees of freedom to get rid of their entropy. However, should that be the case, $\mathrm{VO}_{2}$ would most likely remain insulating even above $T_{c}$, which is not the case, all the more so because $k_{B} T_{c}$ is more than one order of magnitude smaller than the optical gap in the M1 phase [52]. For the same reason, we must exclude a transition merely driven by the larger electronic entropy of the metal.

We are thus inclined to believe that the structural distortion is also necessary to stabilize the insulating phase in $\mathrm{VO}_{2}$, but, once again, not sufficient in view of the behavior of the M2 phase, and of the bad metal character of the R phase [53-55]. It is therefore quite likely that Goodenough's scenario is after all correct, though it requires an active contribution from electronic correlations.

Indeed, different density functional theory (DFT)-based calculations, which should properly account for the effects of the lattice distortion on the electronic structure, though within an independent-particle scheme, do not agree one with another, and none explains at once all experiments. For instance, straight local density approximation (LDA) or generalized gradient approximation (GGA) methods do not find any gap opening in M1 and M2 phases [56,57]. Such gap is instead recovered by GW [58-60] or LDA + U [61-63], in all its variants. However, GW does not give easy access to the total energy, and therefore it does not explain why low temperatures should favor the M1 distorted phase against the rutile undistorted one. In turns, LDA + U or GGA + U calculations, known to overemphasize the exchange splitting, predict the existence of local moments even in the rutile phase [61-63], not observed in experiments [64]. Relatively recent calculations based on Heyd-Scuseria-Ernzerhof hybrid functionals bring even worst results: both rutile and M1 phases are predicted to be magnetically ordered insulators, with the former lower in energy $[65,66]$, even though earlier calculations were claimed to be more in accordance with experiments [25]. In turn, modified Becke and Johnson (mBJ) exchange potentials seem to predict the proper conducting behavior of the R and M1 phases, as well as their lack of magnetism [67], which is erroneously predicted to occur also in the M2 phase [63]. This suggests that suppression of magnetic moments is somehow the rule of $\mathrm{mBJ}$ functionals applied to $\mathrm{VO}_{2}$, which only by chance is the correct result for $\mathrm{R}$ and $\mathrm{M} 1$ phases. Finally, calculations based on the hybrid functional PBE0, that mixes Perdew-Burke-Ernzerhof functional and Hartree-Fock exchange, properly account for the magnetic and electronic properties of M1 and M2 phases, but predict ferromagnetism in the rutile structure, at odds with experiments [33], as well as the existence of a never observed ferromagnetic and insulating monoclinic phase, dubbed M0 [68], also predicted by PerdewBurke-Ernzerhof functionals designed for solids [69].

One might expect that combining ab initio techniques with many-body tools, e.g., DFT with dynamical mean-field theory (DMFT) [70], should work better and finally provide uncontroversial results in accordance with experiments. Unfortunately, different calculations by state-of-the-art DFT + DMFT methods do not even agree about an unanimous view of the M1 monoclinic phase. Specifically, M1 has been regarded from time to time as a correlation-assisted Peierls insulator [24,71], or, vice versa, as a Peierls-assisted Mott insulator [72], or, finally, as a genuine Mott insulator [26,73,74].

In view of the above controversial results, we think it is worth desisting from describing $\mathrm{VO}_{2}$ straight from first principles and rather focusing on a minimal model, which can include all the ingredients that are, by now, widely accepted to be essential. As we mentioned, electron-electron correlations must play an important role and thus need to be included and handled in a truly many-body scheme. At the same time, the coupling of the electrons to the lattice is equally important and must be included as well. We earlier mentioned that the monoclinic distortion in the M1 phase actually entails two different antiferrodistortive components: the basal-plane displacement of $\mathrm{V}$ from the octahedron center, resulting in a zigzag shape of the formerly straight chains, and the outof-plane displacement that produces the chain dimerization. The two phenomena may actually occur separately, as indeed proposed by Goodenough [38], who argued that, generically, the basal-plane distortion should appear at higher temperatures than dimerization. Indeed, time-resolved spectroscopy 
measurements during a photoinduced monoclinic-to-rutile transition have shown that dimerization melts on earlier timescales than the basal-plane displacement [37,75,76], which therefore must be distinct and actually more robust than the former. We must mention, however, that this conclusion does not agree with other experiments [77-80]. More convincing evidence is offered by the monoclinic metal that intrudes, under equilibrium conditions, between rutile metal and monoclinic insulator at ambient pressure [81-84], not just above a critical pressure as originally believed [85]. This phase might correspond to a crystal structure where dimerization is almost melted unlike the zigzag distortion [69,83], so that $e_{g}^{\pi}$ are still above the $a_{1 g}$, though the dimerization is too weak to stabilize at that temperature and hybridization gap within the $a_{1 g}$ band [27]. Even the disappearance prior to the metal-insulator transition [86] of the so-called singlet peak, which is associated to dimerization and observed in optics, can be regarded as a consequence of the melting of dimerization preceding the complete monoclinic-to-rutile transformation. All the above experimental facts point to the need to treat separately the basal-plane displacement and the out-of-plane one. Finally, the importance of the basal plane antiferrodistortive mode suggests the last ingredient to be considered: the multiorbital physics. This aspect was originally emphasized by Goodenough [38] and successively confirmed by many optical measurements [52,87,88].

To summarize, we shall consider a microscopic model which includes the following relevant features:

(1) the electron-electron correlations and the coupling to the lattice distortion [46,53,89-110];

(2) the existence of two different antiferrodistortive components, each playing its own distinctive role [37,38,75]; and

(3) the multiorbital physics [38,52,87,88],

with the minimal requirement of capturing, at least at a qualitative level, the following aspects of the $\mathrm{VO}_{2}$ physics:

A. the existence of an undistorted paramagnetic metal and a monoclinic distorted insulator [43,111-114];

B. the first-order character of the transition between them $[18,115-125]$; and

C. the possible existence of an intermediate monoclinic metal [81-83,126-134].

Many models have been already put forth to describe $\mathrm{VO}_{2}$. However, most of them focus either on the role of the electron-electron correlations or on that of the electron-lattice coupling [27,29,135-148], and thus do not allow access in a single framework to the whole $\mathrm{VO}_{2}$ phase diagram, e.g., the points $\mathrm{A}, \mathrm{B}$, and $\mathrm{C}$ above. Despite that, we must mention that the purely electronic dimer Hubbard Model presented in Ref. [27], which by construction is not able to capture the monoclinic to rutile phase transition, is nevertheless able to describe some of the observed features of the monoclinic metal, like the MIR peak in the optical conductivity observed in Ref. [54]. There are actually some exceptions where electron-electron and electron-lattice interactions have been considered on equal footing [149-151]. In particular, the model studied in Ref. [150] includes explicitly all ingredients listed above. However, therein it is assumed a small bandwidth of the $a_{1 g}$-derived band as compared to the $e_{g}^{\pi}$ one, which contradicts LDA calculations [56]. Moreover, Ref. [150] includes the two distinct effects of the monoclinic distortion, but parametrized by a single displacement variable. In this way, they preclude the possibility to describe the emergence of the monoclinic metal that seems to be observed experimentally. Furthermore, the mean-field treatment of the electron-electron interaction, despite its strength being comparable to the conduction bandwidth, yields not surprisingly to the formation of local moments in the rutile metal, not in accordance with magnetic measurements [64]. This negative result, highlighted by the same authors of Ref. [150], solicits for a more rigorous treatment of the interaction.

This is actually the scope of the present work, which is organized as follows. In Sec. II, we introduce a simple model that includes the three ingredients previously outlined, which we believe should capture the main physics of vanadium dioxide. In Sec. III, we discuss the dynamical mean-field theory (DMFT) approach to the model Hamiltonian, and present in Sec. III its ground-state phase diagram. In Sec. IV, we discuss the insulator-metal transition that occurs in our model upon raising the temperature. In Sec. IV A, we discuss the case in which such transition is driven solely by the electronic entropy, hence neglecting the lattice contribution to entropy, whereas in Sec. IV B we show the opposite case. We will show that the latter situation is rather suggestive, since it foresees different transition temperatures of the two antiferrodistortive components, as predicted by Goodenough [38]. In turn, this result might explain the evidence supporting the existence of a monoclinic metal phase. Finally, Sec. V is devoted to concluding remarks.

\section{THE MODEL}

As we mentioned, the orbitals that are relevant to describe the physics of $\mathrm{VO}_{2}$ are the vanadium $3 d-t_{2 g}$ ones, comprising the $a_{1 g}$ singlet and $e_{g}^{\pi}$ doublet, which host a single conduction electron. We believe that in this circumstance the doublet nature of the $e_{g}^{\pi}$ is not truly essential; what really matters is the distinction between $a_{1 g}$ and $e_{g}^{\pi}$ based on their bonding character with the ligands and response to atomic displacement. Therefore, in order to simplify our modeling without spoiling the important physics, we shall associate the $e_{g}^{\pi}$ doublet with just a single orbital [136,152], which, together with the other orbital mimicking the $a_{1 g}$ singlet, give rise to two bands, band $1 \leftrightarrow a_{1 g}$ and band $2 \leftrightarrow e_{g}^{\pi}$, which accommodate one electron per site; i.e., they are quarter filled.

The other ingredient that is necessary to properly describe $\mathrm{VO}_{2}$ is the electron-electron Coulomb interaction. However, since the main role that Coulomb repulsion is believed to play is to suppress charge fluctuations on $V^{4+}$, we shall ignore the long-range tail and replace Coulomb repulsion with a shortrange interaction.

Finally, we need to include the coupling to the lattice. For simplicity, we shall focus our attention only on the rutile and monoclinic M1 phases, as such ignoring the M2 phase, which is actually regarded by some as just a metastable modification of the M1 structure [40,44,146]. Under this assumption, we can model the lattice antiferrodistortion through a two-component zone boundary mode at momentum $\mathbf{Q}$, with displacement $\mathbf{X}=\left(X_{1}, X_{2}\right)$ and classical potential energy $\Phi\left(X_{1}, X_{2}\right)$. The $X_{1}$ and $X_{2}$ components model, respectively, the 
dimerizing out-of-plane displacement and the band-splitting basal-plane one; see Fig. 1 [135,153].

The model Hamiltonian is thus written as the sum of three terms:

$$
\mathcal{H}=\mathcal{H}_{\mathrm{el}}+\Phi\left(X_{1}, X_{2}\right)+\mathcal{H}_{\mathrm{el}-\mathbf{x}}
$$

$\mathcal{H}_{e l}$ is the purely electronic component reading

$$
\mathcal{H}_{\mathrm{el}}=\sum_{a=1}^{2} \sum_{\mathbf{k}}\left(\epsilon_{a \mathbf{k}}-\mu\right) n_{a \mathbf{k}}+\frac{U}{2} \sum_{i} n_{i}\left(n_{i}-1\right),
$$

where $n_{a, \mathbf{k}}$ is the occupation number at momentum $\mathbf{k}$ of the band $a=1,2, n_{i}$ is the electron number operator at site $i, \mu$ is the chemical potential used to enforce the quarter filling condition and, finally, $U$ is the on-site Hubbard repulsion.

With the aim to reduce the number of independent Hamiltonian parameters, we assume that the density-of-states (DOS) $\mathcal{D}_{1}(\epsilon)$ and $\mathcal{D}_{2}(\epsilon)$, of bands 1 and 2 , respectively, have the same bandwidth and center of gravity, which we shall take as the zero of energy. In addition, we consider both DOS symmetric with respect to their center, and such that $\epsilon_{1 \mathbf{k}}=-\epsilon_{1 \mathbf{k}+\mathbf{Q}}$, where $\mathbf{Q}$ is the wave vector of the antiferrodistortive mode $\mathbf{X}$. This assumption actually overestimates the dimerization strength, since it entails that any $X_{1} \neq 0$ is able to open a hybridization gap in the middle of band 1 , which, we remark, does not coincide with the chemical potential unless band 2 is pushed above it. This implies that a finite hybridization gap within band 1 does not stabilize an insulator so long as band 2 still crosses the Fermi energy. Therefore, our simplified modeling does not spoil the important feature that a distorted insulating phase may occur only above a critical threshold of the Hamiltonian parameters, although it affects the value of that threshold, whose precise determination is, however, behind the scope of the present study.

In order to emphasize the bonding character of the $a_{1 g}$, band 1, along the $c_{R}$ axis, as opposed to the more isotropic $e_{g}^{\pi}$, band 2 , we choose the following forms of the two corresponding DOS's:

$$
\begin{aligned}
& \mathcal{D}_{1}(\epsilon)=\frac{1}{\mathcal{N}}\left[a \epsilon^{2}-b \epsilon^{4}+D^{2}\left(b D^{2}-a\right)\right], \\
& \mathcal{D}_{2}(\epsilon)=\frac{2}{\pi D} \sqrt{1-\left(\frac{\epsilon}{D}\right)^{2}},
\end{aligned}
$$

with $\epsilon \in[-D, D]$ and $\mathcal{N}$ being a normalization factor. We take $b>a / D^{2}>0$ so that $\mathcal{D}_{1}(\epsilon)$ has a double-peak structure evocative of a one-dimensional DOS [71,150,154]. Hereafter, we take the half bandwidth $D=1$ as our energy unit and fix $a D^{3}=1.9$ and $b D^{5}=2.1$. The resulting DOS's are shown in Figs. 2(a) and 2(b). There we note the two-peak structure of the band 1 DOS, mimicking the Van Hove singularities of a quasi-one-dimensional band structure, in contrast to the structureless band 2 DOS.

We highlight that the electron-electron interaction in Eq. (2) only includes the monopole Slater integral $U>0$ and not higher order multipoles responsible of Hund's rules. This approximation, which makes the analysis more transparent, requires some justification. The Coulomb interaction of a single vanadium projected onto the $t_{2 g}$ manifold, which effectively behaves as an $l=1$ atomic shell, can be written in

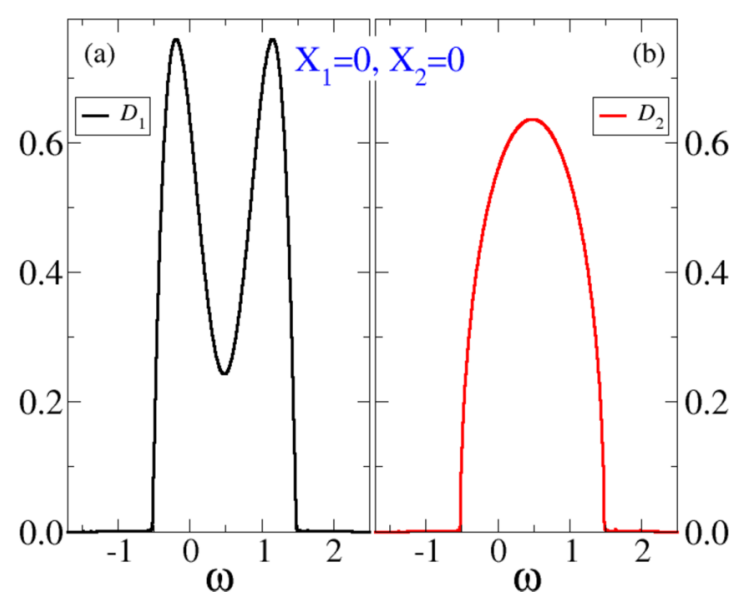

FIG. 2. The density of states $\mathcal{D}_{a}(\omega), \mathrm{a}=1,2$ for the two orbitals for $U=0, X_{1}=0$, and $X_{2}=0$.

terms of two Slater integrals as

$$
\mathcal{H}_{\mathrm{t}_{2 g}}=\frac{U_{\mathrm{t}_{2 g}}-3 J_{\mathrm{t}_{2 g}}}{2} n^{2}-\frac{J_{\mathrm{t}_{2 g}}}{2}[4 S(S+1)+L(L+1)],
$$

where $n, S$, and $L$ are the total occupation, spin, and angular momentum, respectively. Common values of the parameters are $U_{\mathrm{t}_{2 g}} \simeq 4 \mathrm{eV}$ and $J_{\mathrm{t}_{2 g}} \simeq 0.68 \mathrm{eV} \simeq U_{\mathrm{t}_{2 g}} / 6$ [24]. Denoting as $E_{0}(n)$ the lowest energy with $n$ electrons in the $t_{2 g}$ shell, the effective Hubbard $U$ for $\mathrm{V}^{4+}$ can be defined through

$$
U=E_{0}(0)+E_{0}(2)-2 E_{0}(1)=U_{\mathrm{t}_{2 g}}-3 J_{\mathrm{t}_{2 g}} \simeq 1.96 \mathrm{eV},
$$

to be compared with the $\mathrm{VO}_{2}$ bandwidth of about $2.6 \mathrm{eV}$ [56]. In units of the half-bandwidth, $U \simeq 1.5$, the value we shall use hereafter $[155,156]$. We observe that the Coulomb exchange $J_{t_{2 g}}$ has no effect on the configurations with $n=0,1$, while it splits those with $n=2$ in three multiplets, with $(S, L)=$ $(0,0),(1,1),(0,2)$, which are spread out over an energy $\simeq$ $J_{t_{2 g}}$, about a quarter of the full bandwidth. Such small value is not expected to qualitatively alter the physical behaviour (see, e.g., Ref. [157]), which justifies our neglect of the exchange splitting in the model Hamiltonian (2).

We model the potential energy $\Phi\left(X_{1}, X_{2}\right)$ using a Landau functional for improper ferroelectrics [56,75,158] expanded up to the sixth order in the lattice displacements:

$$
\begin{aligned}
\Phi\left(X_{1}, X_{2}\right)= & N\left[\frac{\alpha}{2}\left(X_{1}^{2}+X_{2}^{2}\right)+\frac{\beta_{1}}{4}\left(2 X_{1} X_{2}\right)^{2}\right. \\
& \left.+\frac{\beta_{2}}{4}\left(X_{1}^{2}-X_{2}^{2}\right)^{2}+\frac{\gamma}{6}\left(X_{1}^{2}+X_{2}^{2}\right)^{3}\right],
\end{aligned}
$$

where $N$ is the number of sites and the couplings $\alpha$ to $\gamma$ are all positive. The terms proportional to $\alpha$, i.e., the harmonic part of the potential, and that proportional to $\gamma$ have full rotational symmetry in the $X_{1}-X_{2}$ plane. On the contrary, $\beta_{1}$ favors a lattice distortion only along one of the two components, whereas $\beta_{2}$ favors a distortion with $\left|X_{1}\right|=\left|X_{2}\right|$. In the specific case of $\mathrm{VO}_{2}, \beta_{2}>\beta_{1}$, and thus it is preferable to equally displace both modes [75] rather than just one of them. 
Finally, we write the electron-lattice coupling as

$$
\begin{aligned}
\mathcal{H}_{\mathrm{el}-\mathbf{X}}= & \mathcal{H}_{\mathrm{el}-\mathbf{X}}\left[X_{1}, X_{2}\right] \\
= & -\frac{g}{2} X_{1} \sum_{\mathbf{k} \sigma}\left(c_{1 \mathbf{k} \sigma}^{\dagger} c_{1 \mathbf{k}+\mathbf{Q} \sigma}+\text { H.c. }\right) \\
& -\frac{\delta}{2} X_{2}^{2} \sum_{\mathbf{k}}\left(n_{1 \mathbf{k}}-n_{2 \mathbf{k}}\right),
\end{aligned}
$$

where $c_{1 \mathbf{k} \sigma}$ creates an electron at momentum $\mathbf{k}$ in orbital 1 with $\operatorname{spin} \sigma$, and we recall that, by construction, $\epsilon_{1 \mathbf{k}}=$ $-\epsilon_{1 \mathbf{k}+\mathbf{Q}}$. The dimerization induced by the out-of-plane displacement $X_{1}$ is controlled by the coupling constant $g$, while $\delta$ parametrizes the strength of the crystal field splitting generated by the basal-plane displacement $X_{2}$. By symmetry, the coupling between the field $X_{1}$ and the electron dimerization is at leading order linear $[135,151]$. The quadratic coupling in $X_{2}$ is intentional and has a physical explanation. Indeed, $X_{2}$ corresponds to the vanadium displacement parallel to the diagonal of the rutile basal plane away from the center of the oxygen octahedron. As a result, the hybridization between the $e_{g}^{\pi}$ and the oxygen ligands closer to the new vanadium position increases, whereas the hybridization with the further oxygen diminishes [150]. At linear order in the V-displacement $X_{2}$, the two opposite variations of the hybridization cancel each other, but, at second order, they add up to a net rise in energy of the $e_{g}^{\pi}$ level; hence the expression in Eq. (7). The Hamiltonian Eq. (1) is invariant under the transformations $X_{1 / 2} \rightarrow-X_{1 / 2}$, reflecting a $\mathrm{Z}_{2} \times \mathrm{Z}_{2}$ (also known as $K_{4}$ or "Vierergruppe") symmetry.

Despite the great simplification effort, the model Hamiltonian Eq. (1) has still several parameters to be fixed. We emphasize that our main goal is to reproduce qualitatively the physics of $\mathrm{VO}_{2}$, without any ambition of getting also a quantitative agreement. Nonetheless, just to be sure we do not to explore a Hamiltonian parameter space completely detached from the real $\mathrm{VO}_{2}$ compound, we choose parameters in line with the existing literature. We already mentioned our choice of $U=1.5$, in units of the half-bandwidth, which is in line with the value used in realistic calculations [24,48,150,159161]. The other parameters involve the phonon variables. We shall choose $g=0.4, \delta=0.2, \alpha=0.155, \beta_{1}=1.75 \times 10^{-3}$, $\beta_{2}=2 \beta_{1}$, and $\gamma=6.722 \times 10^{-4}$. Those values permit to reproduce the interband character of the band gap experimentally observed for the monoclinic insulator [87] and to obtain a size of it close enough to the experimental findings; see Sec. III for further details on the spectral properties of the system. Moreover, we checked a posteriori that we can reasonably reproduce the size of the electron-phonon interaction $[136,162,163]$ and the lattice energy change across the rutile-to-monoclinic transition [163] as they were obtained in previous experiments or theoretical analysis. As a concluding remark, we point out that the direct experimental fits of the coupling constants is satisfactorily in agreement with previous estimations of the same [75,164], further corroborating our choice of the parameter set.

\section{DMFT SOLUTION}

We solve the model Hamiltonian Eq. (1) by means of DMFT [165] within the adiabatic Born-Oppenheimer approximation. This approach will allow us to treat correlation effects nonperturbatively beyond an independent-particle description. Exploiting the Born-Oppenheimer approximation, we solve the electronic problem at a fixed displacement $\mathbf{X}=$ $\left(X_{1}, X_{2}\right)$. To any choice of the displacement $\mathbf{X}$ it corresponds to a different electronic problem through the electron-phonon coupling discussed above. Within DMFT, such resulting interacting lattice electrons problem is mapped onto a quantum impurity model constrained by a self-consistency condition, which aims to determine the bath so to describe the local physics of the lattice model. The effective bath is described by a frequency-dependent Weiss field $\mathcal{G}_{0, a}\left(i \omega_{n}\right)$, with $a=$ 1,2 the orbital index. The self-consistency condition relates the Weiss fields to the local self-energy function $\Sigma_{a}\left(i \omega_{n}\right)$, obtained from the solution of the effective quantum impurity model, and the local interacting Green's function

$$
G_{\mathrm{loc}, a}\left(i \omega_{n}\right)=\int_{\mathbb{R}} d \varepsilon \mathcal{D}_{a}(\varepsilon) \frac{1}{\zeta_{a}-\varepsilon},
$$

where $\zeta_{a}=i \omega_{n}+\mu-\Sigma_{a}\left(i \omega_{n}\right)$. The self-consistency conditions read

$$
\mathcal{G}_{0, a}^{-1}\left(i \omega_{n}\right)=G_{\mathrm{loc}, a}^{-1}\left(i \omega_{n}\right)+\Sigma_{a}\left(i \omega_{n}\right) .
$$

Once a Weiss field $\mathcal{G}_{0, a}\left(i \omega_{n}\right)$ is given, the solution to the DMFT equations is obtained iteratively as follows. We solve the effective quantum impurity problem associated to the given Weiss field using exact diagonalization technique $[166,167]$. To this end, we discretize the effective bath into a number $N_{b}$ of electronic levels [166-168]. The resulting Hamiltonian is diagonalized using Lanczos method and the ground-state (at zero temperature) or low-lying states in the spectrum (at finite temperature) are determined [168]. The impurity Green's functions $G_{a}\left(i \omega_{n}\right)$ are then obtained using the dynamical Lanczos technique [165,168]. The selfenergy is obtained by solving the Dyson equation for the impurity problem $\Sigma_{a}\left(i \omega_{n}\right)=\mathcal{G}_{0, a}^{-1}\left(i \omega_{n}\right)-G_{a}^{-1}\left(i \omega_{n}\right)$. The selfenergy is used to evaluate the local interacting Green's function and, finally, to update the Weiss fields by means of the self-consistency condition. The procedure is iterated until the overall error on the determination of the Weiss field falls below a threshold, which in our calculations was set to $10^{-6}$. In this work, we use $N_{b}=8$ as the total number of bath sites, corresponding to a finite system of $N=10$ electronic levels or $N_{s}=20$ spins. Yet, we tested our results with respect to larger values of $N_{b}$ without finding significant differences.

Using the DMFT method, we computed the electronic properties for several values of the displacement $\mathbf{X}=$ $\left(X_{1}, X_{2}\right)$. The part of Eq. (7) related to the tilting $X_{2}$ enters in the single-particle term of the impurity Hamiltonian as the usual crystal field splitting, while the dimerization $X_{1}$ acts directly on the density of states of band 1 [see the expression of $\mathcal{D}_{1}(\epsilon)$ appearing in Eq. (3)], and it opens a gap of size $2 g X_{1}$ in correspondence to its center of gravity.

We calculate the total electronic energy, or the free energy at finite temperature, which renormalizes the Born-Oppenheimer adiabatic potential of the displacement $\Phi\left(X_{1}, X_{2}\right) \rightarrow \Phi_{\text {eff }}\left(X_{1}, X_{2}\right)$ through

$$
\Phi_{\text {eff }}\left(X_{1}, X_{2}\right)=\Phi\left(X_{1}, X_{2}\right)+\left\langle H_{\mathrm{el}}\right\rangle+\left\langle H_{\mathrm{el}-\mathbf{X}}\right\rangle .
$$

We shall restrict our analysis to the paramagnetic sector forcing spin $S U$ (2) symmetry. However, we did check that 
magnetic solutions are higher in energy. We first present results at zero temperature $T=0$, and then move to those at $T>0$.

\section{Ground-state phase diagram}

In Fig. 3(a), we show the adiabatic potential $\Phi_{\text {eff }}\left(X_{1}, X_{2}\right)$ in (8) calculated by DMFT at $U=1.5$. The energy landscape shows five minima. A local minima is located at the origin $X_{1}=X_{2}=0$, and corresponds to an undistorted metal that we identify with the $\mathrm{R}$ phase of vanadium dioxide. Four degenerate global minima are instead located at $X_{1} \simeq \pm 1.5$ and $X_{2} \simeq \pm 2.1$, which are related to each other by the $\mathrm{Z}_{2} \times \mathrm{Z}_{2}$ symmetry and represent the four equivalent lattice distortions. We find that these global minima describe an insulating phase, and thus realize a two-band version of the Goodenough scenario [38] for the M1 phase, in qualitative agreement with $a b$ initio calculations of $\mathrm{VO}_{2}[146,147]$. A detailed discussion of the electronic properties of all minima is postponed to Sec. III.

In Figs. 3(b) and 3(c), we instead show the evolution of the adiabatic potential $\Phi_{\text {eff }}\left(X_{1}, X_{2}\right)$ along some specific lines, as indicated in Fig. 3(a). We note that along the horizontal and vertical cuts, marked by a diamond and a circle in Fig. 3(a), respectively, the energy landscape shows a saddle point, i.e., a minimum along the cut direction but maximum in the perpendicular one. Within our model description, the effect of a uniaxial tensile strain would be taken into account by adding to the Hamiltonian Eq. (1) terms like $-F_{1} X_{1}^{2}$ or $-F_{2} X_{2}^{2}$ $\left(F_{1}, F_{2}>0\right)$, depending on the direction of the applied stress [169-171]. In the presence of such terms, the saddle points observed in Fig. 3(a) along the lines $X_{1}=0$ or $X_{2}=0$ may turn into additional minima of the energy landscape [148], which can possibly describe the occurrence of the M2 phase in the framework of the same model Hamiltonian.

In order to understand the role of the Hubbard interaction $U$ in stabilizing the insulating solution, we studied the evolution of $\Phi_{\text {eff }}\left(X_{1}, X_{2}\right)$ for several values of $U$, along the line in the $X_{1}-X_{2}$ plane connecting the rutile local minimum with one of the monoclinic global minima [the diagonal cut in Fig. 3(a) marked by a diamond symbol]. Our results are reported in Fig. 4. We note that already at $U=0$ the energy has two minima. One is at the origin and corresponds to the undistorted metal. The other is located at finite $\mathbf{X}$ and thus represents a distorted phase that must evidently be also insulating in order to be a local energy minimum. Therefore, at small $U \lesssim 0.2$, the stable phase is the undistorted metal at $X=0$ in Fig. 4, while the local minimum at $X \neq$ 0 (monoclinic insulator) is metastable. However, for larger $U \gtrsim 0.2$, the situation is reversed: The distorted insulator becomes the global minimum, while the undistorted metal is a local one, entailing the typical scenario of a first-order metalinsulator transition driven by interaction. The above results show that electron-electron interaction is crucial to stabilize the distorted insulator, though the active contribution of the lattice is equally essential. Indeed, the interaction strength, $U \simeq 1.5$, the half-bandwidth, is too small to drive on its own the metal-insulator transition [157]. In other words, the picture that emerges from Fig. 4, with the interaction and the coupling to the lattice both necessary to stabilize the insulator, fully confirm our expectation in Sec. I.
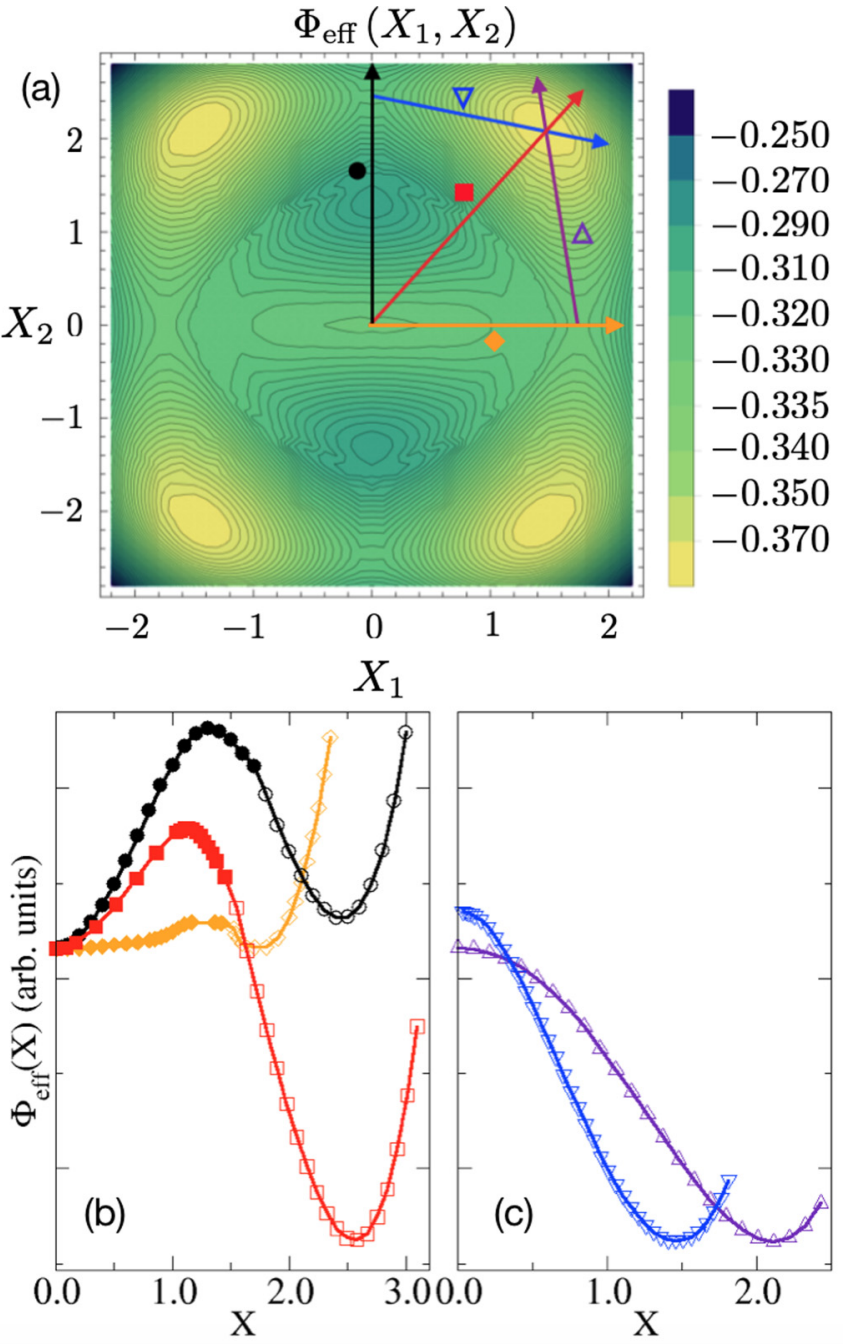

FIG. 3. (a) The zero-temperature color map of the internal energy of the system as function of the amplitude of the crystal distortions $X_{1}$ and $X_{2}$ for $U=1.5$. The system displays five minima, one at $X_{1}=X_{2}=0$ corresponding to a metallic undistorted phase, the others at $X_{1} \simeq \pm 1.5$ and $X_{2} \simeq \pm 2.1$ corresponding to four equivalent insulating and distorted states. [(b), (c)] Evolution of the zerotemperature internal energy along the paths shown in panel (a), where the symbols close to them correspond to the ones used in panels (b) or (c); the coordinate $X=\sqrt{X_{1}^{2}+X_{2}^{2}}$ is computed along the lines as depicted in panel (a). Filled symbols correspond to a two-band metallic solution, whereas empty symbols correspond to an insulating solution everywhere except for the black curve with the circles, where they correspond to a single-band metallic phase. Particularly, in panel (b), the circles (diamonds) correspond to the evolution of the internal energy along the line that involves just the distortion $X_{2}\left(X_{1}\right)$ and the squares correspond to the line that connects the undistorted metal and the distorted insulator found in the $X_{1}, X_{2}>0$ sector. In panel (c), the upward-pointing triangles (downward-pointing triangles) correspond to the line that connects the minimum observed in panel (b) along the line with the circles (diamonds) that involves just the distortion $X_{1}\left(X_{2}\right)$ to the global insulating minimum at $\left(X_{1}, X_{2}\right)=(1.5,2.1)$.

\section{Spectral functions}

Further insights into the properties of the metal-insulator transition can be gained by looking at the spectral 


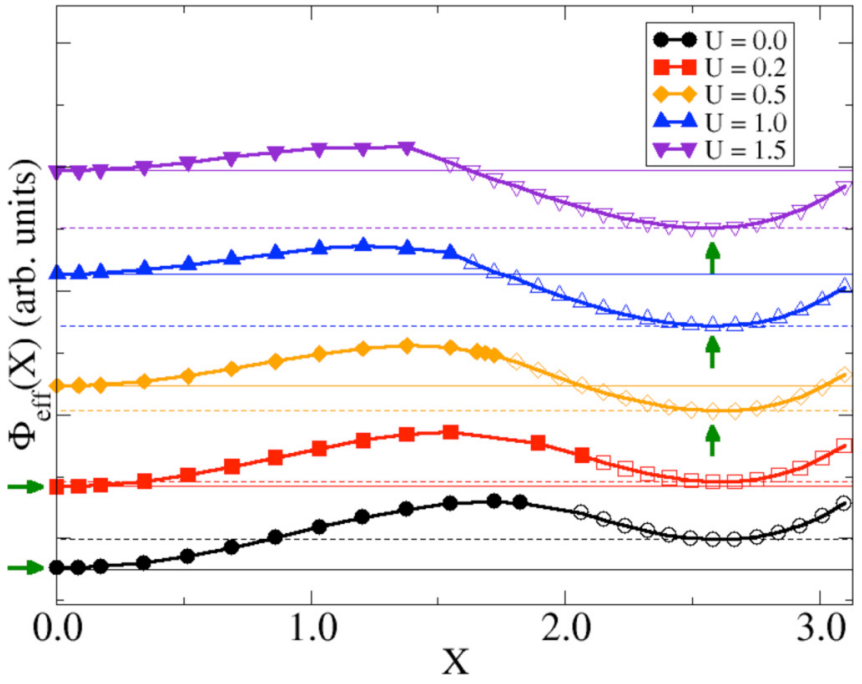

FIG. 4. The zero-temperature internal energy of the system (in arbitrary units) as function of the amplitude of the crystal distortion $X=\sqrt{X_{1}^{2}+X_{2}^{2}}$ (coordinate taken along the line that connects the rutile solution and one of the monoclinic minima) for several values of the Hubbard interaction $U$. Filled (open) symbols correspond to a metallic (insulating) solution. The continuous (dashed) horizontal lines indicate the values of the metallic (insulating) minimum at each value of $U$. Arrows indicate the position of the absolute minimum for each value of the interaction.

functions:

$$
A_{a}(\omega)=-\frac{1}{\pi} \operatorname{Im} G_{\mathrm{loc}, a a}(\omega),
$$

where $a=1,2$ and $G_{\mathrm{loc}, a a}$ is the local interacting Green's function obtained within the DMFT solution of the model. In Fig. 5, we show $A_{a}(\omega)$ at the different minima in Fig. 3(a), with $\omega$ measured with respect to the chemical potential. We note that already in the absence of interaction, $U=0$, the different shapes of the DOS's (see Fig. 2) lead to a larger occupation of band 1 than band 2. Such population unbalance is increased by $U>0$, which effectively enhances the crystal field, leading to an even larger occupation of band 1 at

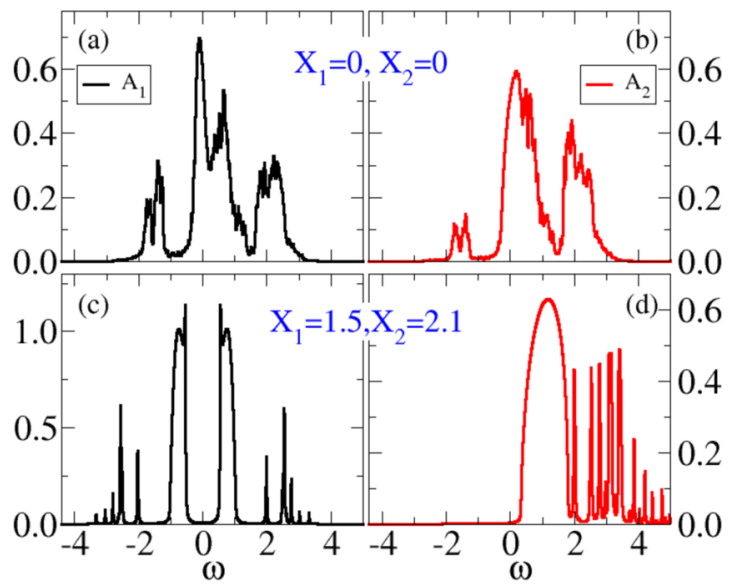

FIG. 5. The spectral functions $A_{a}(\omega), \mathrm{a}=1,2$ for the two minima shown in Fig. 4 at $U=1.50$. The metallic phase corresponds to $X=0[(\mathrm{a}),(\mathrm{b})]$, and the insulator corresponds to $X \sim 2.58$ [(c), (d)]. expenses of $2[152,172,173]$. This is evident in the spectral function of the undistorted metal at $U=1.5$, reported in Figs. 5(a) and 5(b), where the occupied $\omega \leqslant 0$ part of $A_{1}(\omega)$ overwhelms that of $A_{2}(\omega)$ more than in the $U=0$ case of Fig. 2. We also note in Figs. 5(a) and 5(b) side peaks that correspond to the precursors of the Hubbard bands.

The scenario is radically different in the insulating solution; see Figs. 5(c) and 5(d). Here we observe the formation of a hybridization gap opening at the chemical potential inside the band 1. Two coherent-like features flank the gap. Band 2 is instead pushed above the Fermi energy, and therefore is empty. We still observe precursors of the Hubbard sidebands in $A_{1}(\omega)$, as well as signatures of the precursor of the upper Hubbard band in $A_{2}(\omega)$, though rather spiky because of the bath discretization.

We note that in the insulating solution the lowest gap corresponds to transferring one electron from band 1 to band 2 , i.e., from $a_{1 g}$ to $e_{g}^{\pi}$ in the $\mathrm{VO}_{2}$ language, and has a magnitude of about $E_{\text {gap }} \sim 0.8 \mathrm{eV}$, for a realistic value of the half-bandwidth of $1.3 \mathrm{eV}$ [56]. This value of the gap is not too far from the experimental one, $E_{\text {gap }}^{\text {ex }} \sim 0.6-0.7 \mathrm{eV}$ $[35,52,119]$. Therefore, our simplified modeling yields results that are not only qualitatively correct but, rather unexpectedly, also quantitatively not far off the actual ones. The band $1 \rightarrow$ band 1 transition, i.e., $a_{1 g} \rightarrow a_{1 g}$, though being slightly higher in energy, has a much steeper absorption edge since it involves the two coherent peaks in Fig. 5(c), already observed in previous works $[24,26,27,160]$. This result is in loose agreement with $\mathrm{x}$-ray absorption spectroscopy linear dichroism experiments $[86,174]$ that are able to distinguish the two absorption processes.

In order to assess the degree of electronic correlations, we calculate the quasiparticle residue of each band in the undistorted metal phase, defined by

$$
Z_{a}=\left(1-\frac{\partial \operatorname{Re} \Sigma_{a a}(\omega)}{\partial \omega}\right)_{\mid \omega=0}^{-1},
$$

with $a=1,2$. We find that the two bands at $X_{1}=X_{2}=0$ show almost the same value $Z_{1 / 2} \sim 0.67$, not inconsistent with more realistic calculations $[24,72,160,175]$. Such agreement, a priori not guaranteed, gives further support to our simple modeling.

\section{PHASE TRANSITION AT FINITE TEMPERATURE}

Our main scope here, however, is to describe the first-order phase transition upon heating from the low-temperature M1 monoclinic insulator to the high-temperature rutile metal. In general, we can envisage a phase transition primarily driven either by the electron entropy or by the lattice one.

Indeed, we note that the electron free energy of the metal solution, which is metastable at $T=0$, must drop faster upon raising temperature than the insulator free energy since the metal carries more electron entropy than the insulator. This effect alone, that is, ignoring lattice entropy, would be able to drive a first-order transition when insulator and metal free energies cross. On the other hand, since the distorted ground state breaks the $Z_{2} \times Z_{2}$ symmetry of the adiabatic lattice potential $\Phi_{\text {eff }}\left(X_{1}, X_{2}\right)$ in Fig. 3, we might expect such symmetry 


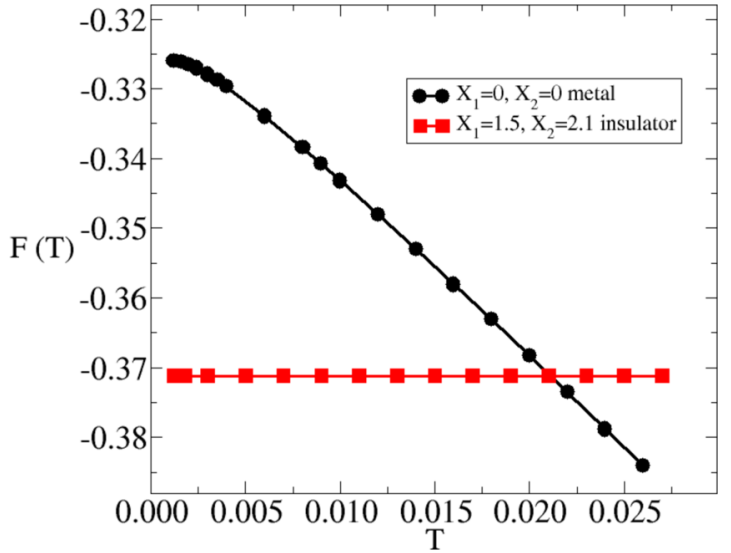

FIG. 6. Temperature evolution of the free energy at the two inequivalent minima $X_{1}=X_{2}=0$ (dots) and $X_{1}=1.5, X_{2}=2.1$ (squares) observed at zero temperature for $U=1.5$. The first-order transition occurs at $T_{\mathrm{el}} \sim 0.021-320 \mathrm{~K}$, of the same order of magnitude as the experimental value $340 \mathrm{~K}$.

to be recovered by raising temperature only because of lattice entropy effects, i.e., ignoring the electronic contribution to entropy.

In reality, both effects should combine to drive the transition. However, dealing together with lattice and electron entropies within our computational scheme would imply to calculate the adiabatic potential $\Phi_{\text {eff }}\left(X_{1}, X_{2}\right)$ at any temperature, which is a rather heavy task. For this reason, in what follows we shall analyze separately electron and lattice entropy effects and at the end argue what would happen should they act together.

\section{A. Electron-driven transition}

Let us first neglect the lattice entropy and study the temperature evolution of the free energies of the two inequivalent minima that we found at zero temperature. For that, we need to evaluate the electronic entropy, which can be obtained through

$$
\begin{aligned}
S\left(X_{1}, X_{2}, T\right) & =\int_{0}^{T} d T^{\prime} \frac{1}{T^{\prime}} \frac{\partial \Phi_{\mathrm{eff}}\left(X_{1}, X_{2}, T^{\prime}\right)}{\partial T^{\prime}} \\
& =\int_{\Phi_{\mathrm{eff}}\left(X_{1}, X_{2}, 0\right)}^{\Phi_{\mathrm{eff}}\left(X_{1}, X_{2}, T\right)} \frac{d \Phi_{\mathrm{eff}}}{T^{\prime}\left(\Phi_{\mathrm{eff}}\right)} .
\end{aligned}
$$

The last equality corresponds to a change of integration variable from the temperature $T^{\prime}$ to the adiabatic potential $\Phi_{\text {eff }}$, which is also the internal energy.

From the entropy $S$ we can estimate the free energy:

$$
F\left(X_{1}, X_{2}, T\right)=\Phi_{\text {eff }}\left(X_{1}, X_{2}, T\right)-T S\left(X_{1}, X_{2}, T\right),
$$

which, we emphasize once more, does not include the lattice contribution to entropy. We shall compare the free energy of the undistorted metal solution at $\mathbf{X}=0$ with that of the distorted insulator at $\mathbf{X} \neq 0$. In principle, the equilibrium displacement in the insulator should change with temperature. In practice, since the entropy of the insulator is negligible for all temperatures under consideration, we shall fix $\mathbf{X}$ at the $T=$ 0 value. The temperature evolution of the metal and insulator free energies so obtained are shown in Fig. 6. As expected, the larger entropy of the metal pushes its free energy below the insulator one at relatively low temperature, $T_{\mathrm{el}} \sim 0.021$, substantially smaller than the insulating gap, and thus justifying our assumption of frozen $\mathbf{X}$. $T_{\mathrm{el}}$ identifies the insulator-metal transition, which is evidently first order since the two free energies cross with different slopes. Incidentally, $T_{\mathrm{el}} \sim 0.021$ in half-bandwidth units corresponds to $\approx 320 \mathrm{~K}$ for a realistic bandwidth of $2.6 \mathrm{eV}$, which has the right order of magnitude when compared with the true critical temperature of $340 \mathrm{~K}$. However, we should mention that a different choice of the parameters appearing in Eq. (1), keeping them still in a regime representative of the physics of vanadium dioxide, would have produced a different value of $T_{\mathrm{el}}$.

\section{B. Lattice-driven transition}

We now move to study the properties of the lattice-driven transition. For that, we first need to model the lattice dynamics. However, since the tetragonal R to monoclinic M1 transition is a complex structural transformation, with martensitic features, especially in films [176-180], our modeling ought to be oversimplified, and aimed just to get qualitatively reasonable results, with no pretension of quantitative accuracy.

As a first step, we must relax our previous assumption of a global antiferrodistortive mode, and instead introduce a displacement field, i.e., a site-dependent displacement $\mathbf{X}_{i}=$ $\left(X_{1 i}, X_{2 i}\right)$. We assume that $\mathbf{X}_{i}$ feels the local adiabatic potential $\Phi_{\text {eff }}\left(\mathbf{X}_{i}\right)$ of Fig. 3(a), temperature independent since we are neglecting the electron entropy. In addition, we suppose that the displacements of nearest-neighbor sites are coupled to each other by an $S O(2) \cong U(1)$ invariant term that tends to minimize the strain. With those assumptions, the classical Hamiltonian reads

$$
\begin{aligned}
\mathcal{H}_{\mathrm{ph}}(\mathbf{X})= & J \sum_{\langle i j\rangle}\left(\mathbf{X}_{i}-\mathbf{X}_{j}\right) \cdot\left(\mathbf{X}_{i}-\mathbf{X}_{j}\right) \\
& +\sum_{i} \Phi_{\mathrm{eff}}\left(\mathbf{X}_{i}\right),
\end{aligned}
$$

where $\mathbf{X}$ denotes a configuration of all the displacement vectors. The model (13) is equivalent to a generalized $X Y$ model, where $\mathbf{X}_{i}$ plays the role of two-component spin of variable length, while $J>0$ is the conventional spin stiffness. $\Phi_{\text {eff }}\left(\mathbf{X}_{i}\right)$ is the effective anisotropic potential obtained from the solution of the electron problem. Both the length and the direction of the local distortion $\mathbf{X}_{i}$ are controlled by the effective potential $\Phi_{\text {eff }}\left(\mathbf{X}_{i}\right)$, which is not invariant under $U(1)$ but under separate $X_{1} \rightarrow-X_{1}$ and $X_{2} \rightarrow-X_{2}$ transformations, i.e., $Z_{2} \times Z_{2}$. The phase diagram of an $X Y$ model in the presence of an anisotropy term that lowers $U(1)$ down to $Z_{n}$ is already known [181-183]. In particular, the anisotropy $Z_{n}$ for $n \geqslant 4$ is a dangerously irrelevant perturbation that does not change the $X Y$ universality class of the transition [182,183]. Our specific case study, where $U(1) \rightarrow Z_{2} \times Z_{2}$, has not been considered yet, at least to our knowledge, but it should most likely change the $X Y$ universality class, which is what we are going to investigate in the following.

We study the classical model Eq. (13) at different temperatures using the standard Monte Carlo (MC) method [184]. We consider the model on a three-dimensional cubic lattice of 
side $N_{x}$. The average value of a given observable, i.e., $\langle O\rangle=$ $\frac{1}{Z} \sum_{\mathbf{X}} \mathcal{O}(\mathbf{X}) e^{-\beta H_{p h}(\mathbf{X})}$, where $Z=\sum_{\mathbf{X}} e^{-\beta H_{p h}(\mathbf{X})}$, is therefore estimated statistically using the MC algorithm to explore the configuration space. New configurations are generated and accepted or rejected using the METROPOLIS algorithm with local updates. Each local update corresponds to a shift $\Delta X_{i}$ of one of the two component $i=1,2$, chosen with equal probability. Within our calculations, we use $\Delta X_{i}=0.15$, yet we checked that smaller values do not change the accuracy of the calculations. In addition, we include the possibility of global moves of the type $\mathbf{X}_{i} \rightarrow\left(-X_{1 i}, X_{2 i}\right),\left(X_{1 i},-X_{2 i}\right)$, or $\left(-X_{1 i},-X_{2 i}\right)$ with a total probability $P_{\text {global }}=0.05$ equally distributed among the three cases, i.e., with probability $P_{\text {global }} / 3$ each. The local updates require the evaluation of the effective potential $\Phi_{\text {eff }}\left(\mathbf{X}_{i}\right)$ at the new value of $\mathbf{X}_{i}$. To speed up execution, we pre-evaluate all the interpolated values of the effective potential at any possible point compatible with the size of the shift using a bicubic spline method. A new configuration of the system is obtained after a full sweep of the lattice sites. The statistical error is thus controlled by the number of sweeps $N_{s}$, to which it corresponds a number $N_{s} N_{x}^{3}$ of MC steps. In order to avoid self-correlation problems, we measure the average of any observables every $N_{\text {meas }} \simeq$ 100 sweeps and in any case after a warmup period of $N_{\mathrm{wp}} \simeq$ 1000 sweeps. In all our calculations, the number of sweeps is of the order of $N_{s}=4-6 \times 10^{5}$. We further minimize the statistical error by executing the numerical computation in parallel with $N_{\text {cpu }}=20 \mathrm{cpu}$. The resulting statistical error is within the symbols in all our plots.

Before discussing the results, we have to mention that some details might depend on the precise form of the coupling between different sites. In the model Hamiltonian, Eq. (13), we have chosen the simplest possible one, i.e., a nearest neighbor coupling, thus disregarding longer range interactions.

In Fig. 7(a), we plot the modulus of the average displacement, $|\langle\mathbf{X}\rangle|$, as function of the temperature. For small system size (e.g., $\left.N_{x}=10\right)|\langle\mathbf{X}\rangle|$ shows a smooth crossover in temperature. However, increasing $N_{x}$ unveils the existence of a continuous phase transition at a critical value $T_{c}$ of the temperature. The actual value of $T_{c}$ depends by the size of the coupling constant $J$ that somehow acts as a unit of measure for the energy. More involved calculations are necessary for the evaluation of $J$ in vanadium dioxide from first-principle calculations and we postpone them for a future work. For that reason, we have preferred to use $T_{c}$ as the unit of temperature in Fig. 7 and in those that follow. In order to better reveal the second-order character of the transition, we also show in Fig. 7(a) the fit with a mean-field square-root behavior. The fit is rather good, although we known that close to the transition the actual critical behavior must deviate from the mean field.

A closer look to the temperature dependence of the order parameter uncovers a nontrivial two-step evolution, which is more evident in Fig. 7(b), where we show the specific heat $C_{v}=\partial\langle E\rangle / \partial T$ vs $T$. Indeed, $C_{v}$ clearly displays two peaks that are suggestive of two distinct transitions. The first transition at $T=T_{c}$, below which $|\langle\mathbf{X}\rangle|$ acquires a finite value, is followed by a second one at lower $T=T_{d}<T_{c}$.

From the knowledge of the specific heat at constant volume $C_{v}(T)$, we can compute the change of the vibrational

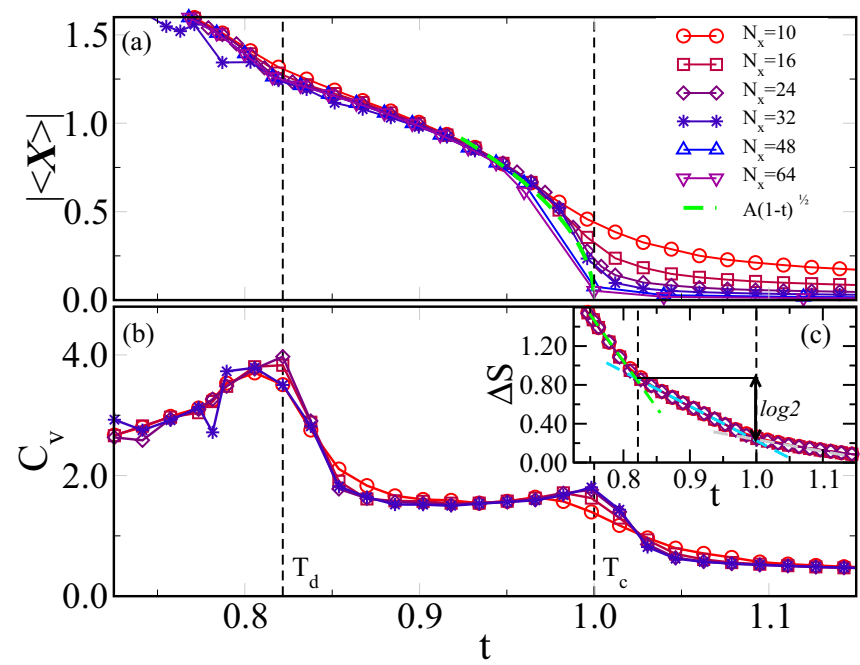

FIG. 7. (a) Modulus of the average displacement as function of the reduced temperature $t=T / T_{c}$. (b) Specific heat for the same model as in panel (a) as function of the reduced temperature. The data are for $N_{s}=4 \times 10^{5} \mathrm{MC}$ sweeps of the lattice and different linear size $N_{x}$ (solid lines and open symbols). The dashed line in the critical region is a fit of the form $A(1-t)^{1 / 2}$, with $A=0.96$. (c) Entropy loss $\Delta S=S_{\infty}-S(t)$ as a function of reduced temperature and $N_{x}$ as in panel (a). $S_{\infty}$ is the value attained in the limit of infinite temperature. The dashed lines are guides to the eye, emphasizing the different linear behaviors of the entropy across the two phase transitions.

entropy in the region where the two transitions occur as $\Delta S(t)=\int_{t}^{\infty} \frac{C_{V}(T)}{T} d T$. This quantity is displayed in Fig. 7(c) and it shows an almost linear behavior in the whole explored temperature range. However, as shown by the dashed lines there, the slopes of the line is different in the three regions $T<T_{d}, T_{d}<T<T_{c}$, and $T>T_{c}$.

In order to understand the nature of both transitions, in Fig. 8 we show at $T>T_{c}$, left panels, $T_{d}<T<T_{c}$, middle panels, and $T<T_{d}$, right panels, the endpoint distribution after $N_{s}=4 \times 10^{5}$ MC sweeps of the lattice of the $N_{x}^{3}$ displacement vectors superimposed to the potential landscape in the $\left(X_{1}, X_{2}\right)$ space (top panels) and a real space snapshot within a single layer of the cubic lattice (bottom panels). At high temperature, $T>T_{c}$, the $\mathbf{X}_{i}$ 's cover homogeneously the whole potential landscape; see Fig. 8 top-left panel; for those without any appreciable spatial correlation, see Fig. 8 bottom-left panel. Lowering $T$ slightly below $T_{c}$, we observe a significant change in the displacement distribution; see Fig. 8 middle panels. Specifically, the system seems to break ergodicity first along $X_{2}$; in the simulation corresponding to the figure it localizes in the $X_{2}>0$ half-plane, while it is still uniform along $X_{1}$. Consequently, clusters of parallel displacement vectors form in real space. The alignment direction has $X_{2}>0$ for all clusters, while the $X_{1}$ component changes from cluster to cluster; see Fig. 8 bottom-middle panel. Only below $T_{d}$ does the full ergodicity breakdown occur, with the system trapped around just one of the four equivalent minima, in the figure with $X_{2}>0$ and $X_{1}>0$. In other words, the $Z_{2} \times Z_{2}$ symmetry of the model Eq. (13) gets broken in two steps upon cooling: First, the $Z_{2}$ symmetry $X_{2} \rightarrow-X_{2}$ spontaneously breaks, and next, the residual $X_{1} \rightarrow-X_{1}$ symmetry 

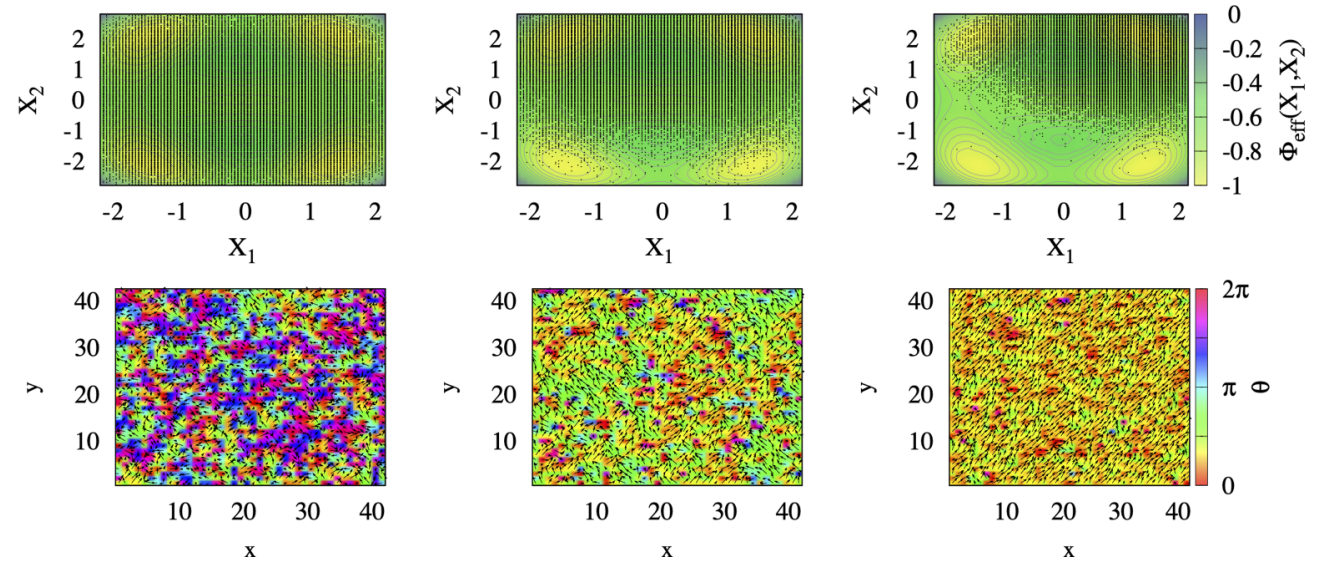

FIG. 8. Top panels: distribution of the displacements $\mathbf{X}_{i}$ at the end of the MC simulation, superimposed to the adiabatic potential $\Phi_{\text {eff }}$, properly normalized so that $\Phi_{\text {eff }} \in[-1,0]$. Data are for $N_{x}=50, N_{s}=4 \times 10^{5} \mathrm{MC}$ sweeps of the lattice, and reduced temperatures $t=T / T_{c}$ : $t=1.03$ (left), 0.82 (center), and 0.71 (right). Each (black) dot represents one of the $N_{x}^{3}$ endpoints of the calculation. Bottom panels: displacement field configuration within a single plane of the cubic lattice, with the same parameters of the top panels. If $\mathbf{X}_{i}=\left|\mathbf{X}_{i}\right|\left(\cos \theta_{i}, \sin \theta_{i}\right)$, the color code represents $\theta_{i} \in[0,2 \pi]$, and the arrow length $\left|\mathbf{X}_{i}\right|$. At high temperature $T>T_{c}$ (left panels) $\mathbf{X}_{i}$ have random length and orientation, thus covering homogeneously the entire potential landscape. For $T_{d}<T<T_{c}$ (center panels), the displacement orientation shows breaking of the $Z_{2}$ symmetry $X_{2} \rightarrow-X_{2}$. At lower temperature $T<T_{d}<T_{c}$, also the residual $Z_{2}$ symmetry $X_{1} \rightarrow-X_{1}$ gets broken; most of the $\mathbf{X}_{i}$ 's have length and direction corresponding to just one of the potential global minima.

breaks, leading to two consecutive Ising-like transitions. This is summarized in Fig. 9, where we see that at $T_{c}\left\langle X_{2}\right\rangle$ becomes finite, and thus also $|\langle\mathbf{X}\rangle|$, while $\left\langle X_{1}\right\rangle$ is still zero. Only below $T_{d}$ does $X_{1}$ acquire a finite average value. Accordingly, the vibrational entropy loss $\Delta S$, shown in Fig. 7(c), changes to a quantity $\sim \ln (2)$ between the two temperatures $T_{d}$ and $T_{c}$. This is consistent with an increase of the available phase space for the system of a factor of 2 by moving from one temperature to the other.

Translated in the language of $\mathrm{VO}_{2}$, these results suggest the existence of an intermediate monoclinic phase for $T_{d}<$ $T<T_{c}$ where the $\mathrm{V}$ atoms are displaced only within the basal plane; i.e., the chains are tilted but not yet dimerized.

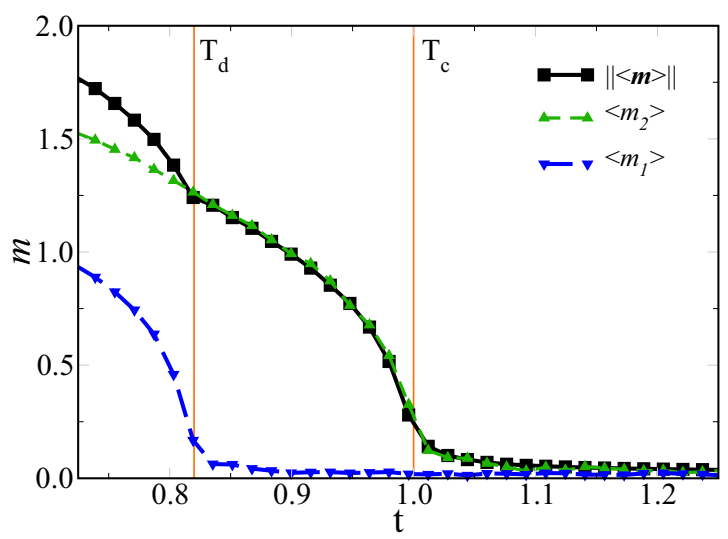

FIG. 9. Magnetization $m$ as a function of the reduced temperature $t=T / T_{c}$. Data are for $N_{s}=6 \times 10^{5} \mathrm{MC}$ sweeps, $N_{x}=24$. The solid line (black with open square) is the modulus of the average magnetization vector. The dashed lines (green and blue with open triangles) indicate the behavior of the average of the magnetization components. The solid (orange) vertical lines indicate the two critical temperatures $T_{d}<T_{c}$ associated to the two-stage transition.
In our model Hamiltonian (1), such phase with $\left\langle X_{1}\right\rangle=0$ describes a monoclinic metal, which, as discussed in Sec. I, has been reported in several experiments [81-83,126-134]. Only below $T_{d}$ are both components of the antiferrodistortive displacement finite, leading to the M1 insulating phase.

In conclusion, without including the electron entropy, we find two transitions that look continuous and in the Ising universality class: one at $T_{d}$ between a monoclinic insulator and a monoclinic metal, and another at $T_{c}>T_{d}$ from the monoclinic metal to a rutile one. In contrast, neglecting the lattice entropy and just including the electronic one, we found in Sec. IV A a single first-order transition at $T_{\mathrm{el}}$, directly from the monoclinic insulator to the rutile metal. We can try now to argue what we could have obtained keeping both entropy contributions still within the Born-Oppenheimer adiabatic approximation.

In that case, we expect that the depth of the rutile minimum in the Born-Oppenheimer potential of Fig. 3 becomes a growing functions of $T$, unlike the depth of the insulating minima, since in the insulator the electronic entropy is negligible with respect to that in the metal. For the same reason, we expect that the height of the two equivalent saddle points at $X_{1}=0$ but $X_{2} \simeq \pm 2.5$ (see the black and blue lines in Figs. 3(b) and 3(c), respectively) lowers with increasing $T$, since these points with large crystal field splitting but without dimerization just describe the monoclinic metal, eventually turning these saddle points into local minima. This effect might well turn the monoclinic-insulator to monoclinic-metal transition at $T_{d}$ into a first order one, all the more if we better modeled the martensitic features of the structural distortion. However, even in that case we still expect a further transition at $T_{c}>T_{d}$ into the rutile metal, unless the latter has such a large entropy compared to the monoclinic metal to drive a first-order transition from the insulator directly into the rutile metal, as it would occur if $T_{\mathrm{el}}<T_{d}$, i.e., if the electronic entropy gain far exceeds the lattice one. 
The experimental evidences supporting the existence of a monoclinic metal phase intruding between the M1 insulator and R metal [81-83,126-134] suggest that, should our modeling be indeed representative of $\mathrm{VO}_{2}$, then the Hamiltonian parameters should be such that $T_{\mathrm{el}} \gtrsim T_{d}$. This also entails a substantial release of lattice entropy across the transition, in accordance with experiments [89,185] and theoretical [186] proposals. We emphasize that $T_{\mathrm{el}} \gtrsim T_{d}$ does not mean that correlations play a minor role, but rather the opposite, since it would imply the insulator, whose internal energy is substantially contributed by electronic correlations, would survive up to much higher temperature if it were not for the lattice.

\section{CONCLUSIONS}

We have constructed a minimal model that we believe contains all essential ingredients to correctly capture the physics of the metal-insulator transition in vanadium dioxide.

The model comprises two orbitals per site, one mimicking the $a_{1 g}$ and the other the $e_{g}^{\pi}$, thus neglecting the twofold nature of the latter, which broaden into two bands. The $a_{1 g}$ band has a double peak structure, reflecting its bonding character along the rutile $c$ axis, while the $e_{g}^{\pi}$ one is structureless. Both have the same bandwidth and center of gravity. The density corresponds to one electron per site; i.e., the two bands are at quarter filling. The electrons feel an on-site Hubbard repulsion and are coupled to two zone-boundary lattice modes, corresponding, respectively, to the basal plane component, i.e., the tilting of the vanadium chains, and out-of-plane component, responsible for the chain dimerization, of the antiferrodistortive displacement that acquires a finite expectation value below the transition from the high-temperature rutile structure to the low-temperature monoclinic one (M1). Using realistic Hamiltonian parameters and assuming the Born-Oppenheimer adiabatic approximation, we find at low temperatures phase coexistence between a stable distorted insulator, the monoclinic M1 insulator, and a metastable undistorted metal, the rutile metal. Upon rising temperature, our model description suggests a two-step transition. First, the dimerization component of the antiferrodistortive displacement melts, leading to a transition from the monoclinic insulator to a monoclinic metal. Second, the tilting component disappears, and the monoclinic metal turns into the rutile one. Such a twotransition scenario, not in disagreement with experiments, is mostly driven by the lattice entropy, also in accordance with experiments.

One of the messages of our model calculation is that the electron-electron interaction has the role to effectively enhance the coupling to the lattice, stabilizing a distorted phase otherwise metastable in the absence of interaction. This also implies that we could have obtained similar results with weaker electronic correlations but stronger electron- lattice coupling. This conclusion is actually supported by the phenomenology of niobium dioxide $\mathrm{NbO}_{2}$, which, mutatis mutandis, is akin to that of $\mathrm{VO}_{2} . \mathrm{NbO}_{2}$ also undergoes a metal-insulator transition, though at substantially higher temperature of $T_{\mathrm{MIT}} \sim 1080 \mathrm{~K}$ [187-190]. There is some experimental evidence of separate structural and electronic phase transitions occurring in this compound [189-192], with a transition temperature for the structural change $T_{\mathrm{s}} \sim 1123 \mathrm{~K}$ [193], from a high-temperature rutile structure to a lowtemperature body-centered tetragonal (BCT) one that locally resembles the $\mathrm{M} 1$ phase of $\mathrm{VO}_{2}$ [191,194-196]. It has been proposed that the mismatch between $T_{\mathrm{MIT}}$ and $T_{\mathrm{S}}$ can be justified by invoking a melting of the dimerization component of the structural distortion in the BCT insulator at smaller temperature as compared to the melting of the tilting component [190-192,197]. The metallic solution that appears in between the two transition temperatures should be mostly metallic along the $c_{R}$ axis, since the almost one-dimensional $a_{1 g}$ band gives the most relevant contribution to the spectral weight at the Fermi level in this intermediate phase. This expectation is not in disagreement with some experimental findings in which they measure, above $T_{\mathrm{MIT}}$, a metallic conductivity along $\mathrm{c}_{R}$ while a semiconducting one in the orthogonal direction [198]. However, we should point out that not all the experiments confirm this scenario [199]. We believe that a similar anisotropy in the conduction properties should be displayed also by the monoclinic metallic phase of vanadium dioxide. The single $4 d$ electron in $\mathrm{Nb}^{4+}$ is expected to be less correlated than the $3 d$ electron in $\mathrm{V}^{4+}$. This loss of correlations, testified by the $\mathrm{VO}_{2} \mathrm{M} 2$ phase having no counterpart in $\mathrm{NbO}_{2}$ [200] and by the efficacy of $a b$ initio methods to describe $\mathrm{NbO}_{2}$ [74,201-203], is actually overcompensated by the increase in covalency due to the broader spatial distribution of the $4 d$ orbitals [204], which, in turn, yields a stronger coupling with the zone-boundary lattice modes and thus a higher transition temperature.

\section{ACKNOWLEDGMENTS}

F.G. thanks Sergiy Lysenko for the useful discussions about the thermodynamic potential for the two phononic modes, as well as for the choice of the parameters appearing there. F.G. thanks also Maja Berović and Daniele Guerci for the discussions concerning the manuscript. A.A. thanks Massimo Capone and Sandro Sorella for useful discussions. We thank Martin Eckstein for fruitful debate. We acknowledge support from the H2020 Framework Programme, under ERC Advanced Grant No. 692670 "FIRSTORM." A.A. also acknowledges financial support from MIUR PRIN 2015 (Prot. No. 2015C5SEJJ001) and SISSA/CNR project "Superconductivity, Ferroelectricity, and Magnetism in Bad Metals" (Prot. No. 232/2015).
[1] Z. Yang, C. Ko, and S. Ramanathan, Oxide electronics utilizing ultrafast metal-insulator transitions, Annu. Rev. Mater. Res. 41, 337 (2011).

[2] T. Driscoll, H.-T. Kim, B.-G. Chae, B.-J. Kim, Y.-W. Lee, N. M. Jokerst, S. Palit, D. R. Smith, M. Di Ventra, and D. N. Basov, Memory metamaterials, Science 325, 1518 (2009).
[3] J. Zhou, Y. Gao, Z. Zhang, H. Luo, C. Cao, Z. Chen, L. Dai, and $\mathrm{X}$. Liu, $\mathrm{VO}_{2}$ thermochromic smart window for energy savings and generation, Sci. Rep. 3, 3029 (2013).

[4] W.-X. Huang, X.-G. Yin, C.-P. Huang, Q.-J. Wang, T.-F. Miao, and Y.-Y. Zhu, Optical switching of a metamaterial by temperature controlling, Appl. Phys. Lett. 96, 261908 (2010). 
[5] Y. Ke, S. Wang, G. Liu, M. Li, T. J. White, and Yi Long, Vanadium dioxide: The multistimuli responsive material and its applications, Small 14, 1802025 (2018).

[6] M. Liu, H. Y. Hwang, H. Tao, A. C. Strikwerda, K. Fan, G. R. Keiser, A. J. Sternbach, K. G. West, S. Kittiwatanakul, J. Lu, S. A. Wolf, F. G. Omenetto, X. Zhang, K. A. Nelson, and R. D. Averitt, Terahertz-field-induced insulator-to-metal transition in vanadium dioxide metamaterial, Nature (London) 487, 345 (2012).

[7] N. I. Zheludev and Y. S. Kivshar, From metamaterials to metadevices, Nat. Mater. 11, 917 (2012).

[8] M. A. Kats, R. Blanchard, S. Zhang, P. Genevet, C. Ko, S. Ramanathan, and F. Capasso, Vanadium Dioxide as a Natural Disordered Metamaterial: Perfect Thermal Emission and Large Broadband Negative Differential Thermal Emittance, Phys. Rev. X 3, 041004 (2013).

[9] J. del Valle, P. Salev, F. Tesler, N. M. Vargas, Y. Kalcheim, P. Wang, J. Trastoy, M.-H. Lee, G. Kassabian, J. G. Ramírez, M. J. Rozenberg, and I. K. Schuller, Subthreshold firing in mott nanodevices, Nature (London) 569, 388 (2019).

[10] C. Zhang, G. Zhou, J. Wu, Y. Tang, Q. Wen, S. Li, J. Han, B. Jin, J. Chen, and P. Wu, Active Control of Terahertz Waves Using Vanadium-Dioxide-Embedded Metamaterials, Phys. Rev. Appl. 11, 054016 (2019).

[11] E. Hoschek and W. Klemm, Weitere beitr age zur kenntnis der vanadinoxyde, Z. Anorg. Allg. Chem. 242, 63 (1939).

[12] O. A. Cook, High-temperature heat contents of $\mathrm{V}_{2} \mathrm{O}_{3}, \mathrm{~V}_{2} \mathrm{O}_{4}$, and $\mathrm{V}_{2} \mathrm{O}_{51}$, J. Am. Chem. Soc. 69, 331 (1947).

[13] G. Andersson, Studies on vanadium oxides. I. Phase analysis, Acta Chem. Scand. 8, 1599 (1954).

[14] M. S. Archer, D. S. P. Roebuck, and F. J. Whitby, Magnetic susceptibility of vanadium dioxide, Nature (London) 174, 754 (1954).

[15] A. Magnéli and G. Andersson, On the $\mathrm{MoO}_{2}$ structure type, Acta Chem. Scand. 9, 1378 (1955).

[16] G. Andersson, Studies on vanadium oxides. II. The crystal structure of vanadium dioxide, Acta Chem. Scand. 10, 623 (1956).

[17] W. Rüdorff, G. Walter, and J. Stadler, Magnetismus, leitfähigkeit und reflexionsspektren von vanadindioxyd und vanadindioxyd-titandioxyd-mischkristallen, Z. Anorg. Allg. Chem. 297, 1 (1958).

[18] F. J. Morin, Oxides Which Show a Metal-to-Insulator Transition at the Neel Temperature, Phys. Rev. Lett. 3, 34 (1959).

[19] S. Westman, Note on a phase transition in $\mathrm{VO}_{2}$, Acta Chem. Scand. 15, 217 (1961).

[20] W. Klemm and L. Grimm, Über die wärmetönung bei der paramagnetischen curie-temperatur"des vanadindioxyds, Naturwissenschaften 27, 787 (1939).

[21] A. X. Gray, M. C. Hoffmann, J. Jeong, N. P. Aetukuri, D. Zhu, H. Y. Hwang, N. C. Brandt, H. Wen, A. J. Sternbach, S. Bonetti, A. H. Reid, R. Kukreja, C. Graves, T. Wang, P. Granitzka, Z. Chen, D. J. Higley, T. Chase, E. Jal, E. Abreu, M. K. Liu, T.-C. Weng, D. Sokaras, D. Nordlund, M. Chollet, R. Alonso-Mori, H. Lemke, J. M. Glownia, M. Trigo, Y. Zhu, H. Ohldag, J. W. Freeland, M. G. Samant, J. Berakdar, R. D. Averitt, K. A. Nelson, S. S. P. Parkin, and H. A. Dürr, Ultrafast terahertz field control of electronic and structural interactions in vanadium dioxide, Phys. Rev. B 98, 045104 (2018).
[22] S. Wall, S. Yang, L. Vidas, M. Chollet, J. M. Glownia, M. Kozina, T. Katayama, T. Henighan, M. Jiang, T. A. Miller, D. A. Reis, L. A. Boatner, O. Delaire, and M. Trigo, Ultrafast disordering of vanadium dimers in photoexcited $\mathrm{VO}_{2}$, Science 362, 572 (2018).

[23] S. Chen, Z. Wang, H. Ren, Y. Chen, W. Yan, C. Wang, B. $\mathrm{Li}$, J. Jiang, and C. Zou, Gate-controlled $\mathrm{VO}_{2}$ phase transition for high-performance smart windows, Sci. Adv. 5, eaav6815 (2019).

[24] S. Biermann, A. Poteryaev, A. I. Lichtenstein, and A. Georges, Dynamical Singlets and Correlation-Assisted Peierls Transition in $\mathrm{VO}_{2}$, Phys. Rev. Lett. 94, 026404 (2005).

[25] V. Eyert, $\mathrm{VO}_{2}$ : A Novel View from Band Theory, Phys. Rev. Lett. 107, 016401 (2011).

[26] W. H. Brito, M. C. O. Aguiar, K. Haule, and G. Kotliar, MetalInsulator Transition in $\mathrm{VO}_{2}$ : A DFT + DMFT Perspective, Phys. Rev. Lett. 117, 056402 (2016).

[27] O. Nájera, M. Civelli, V. Dobrosavljević, and M. J. Rozenberg, Resolving the $\mathrm{VO}_{2}$ controversy: Mott mechanism dominates the insulator-to-metal transition, Phys. Rev. B 95, 035113 (2017).

[28] D. Plašienka, R. Martoňák, and M. C. Newton, Ab initio molecular dynamics study of the structural and electronic transition in $\mathrm{VO}_{2}$, Phys. Rev. B 96, 054111 (2017).

[29] O. Nájera, M. Civelli, V. Dobrosavljević, and M. J. Rozenberg, Multiple crossovers and coherent states in a Mott-Peierls insulator, Phys. Rev. B 97, 045108 (2018).

[30] S. Kim, K. Kim, C.-J. Kang, and B. I. Min, Correlationassisted phonon softening and the orbital-selective Peierls transition in $\mathrm{VO}_{2}$, Phys. Rev. B 87, 195106 (2013).

[31] J. H. Park, J. M. Coy, T. S. Kasirga, C. Huang, Z. Fei, S. Hunter, and D. H. Cobden, Measurement of a solid-state triple point at the metal-insulator transition in $\mathrm{VO}_{2}$, Nature (London) 500, 431 (2013).

[32] Y. Chen, S. Zhang, F. Ke, C. Ko, S. Lee, K. Liu, B. Chen, J. W. Ager, R. Jeanloz, V. Eyert, and J. Wu, Pressure-temperature phase diagram of vanadium dioxide, Nano Lett. 17, 2512 (2017).

[33] J. B. Goodenough, Magnetism and the Chemical Bond, Interscience Monographs on Chemistry: Inorganic Chemistry Section (Interscience Publishers, New York, 1963).

[34] G. Villeneuve and P. Hagenmuller, Metal-insulator transitions in pure and doped $\mathrm{VO}_{2}$, in Localization and Metal-Insulator Transitions, edited by H. Fritzsche and D. Adler (Springer, Boston, MA, 1985), pp. 39-52.

[35] C. N. Berglund and H. J. Guggenheim, Electronic properties of $\mathrm{VO}_{2}$ near the semiconductor-metal transition, Phys. Rev. 185, 1022 (1969).

[36] Y.-R. Jo, M.-W. Kim, and B.-J. Kim, Direct correlation of structural and electrical properties of electron-doped individual $\mathrm{VO}_{2}$ nanowires on devised TEM grids, Nanotechnology 27, 435704 (2016).

[37] P. Baum, D.-S. Yang, and A. H. Zewail, 4d visualization of transitional structures in phase transformations by electron diffraction, Science 318, 788 (2007).

[38] J. B. Goodenough, The two components of the crystallographic transition in $\mathrm{VO}_{2}$, J. Solid State Chem. 3, 490 (1971).

[39] A. Marcelli, M. Coreno, M. Stredansky, W. Xu, C. Zou, L. Fan, W. Chu, S. Wei, A. Cossaro, A. Ricci, A. Bianconi, and 
A. D'Elia, Nanoscale phase separation and lattice complexity in $\mathrm{VO}_{2}$ : The metal-insulator transition investigated by Xanes via auger electron yield at the vanadium $\mathrm{L}_{23}$-edge and resonant photoemission, Condens. Matter 2, 38 (2017).

[40] J. P. Pouget, H. Launois, T. M. Rice, P. Dernier, A. Gossard, G. Villeneuve, and P. Hagenmuller, Dimerization of a linear Heisenberg chain in the insulating phases of $\mathrm{V}_{1-x} \mathrm{Cr}_{x} \mathrm{O}_{2}$, Phys. Rev. B 10, 1801 (1974).

[41] A. Zylbersztejn and N. F. Mott, Metal-insulator transition in vanadium dioxide, Phys. Rev. B 11, 4383 (1975).

[42] J. Pouget and H. Launois, Metal-insulator phase transition in $\mathrm{VO}_{2}$, J. Phys. Colloques 37, C4-49 (1976).

[43] C. N. Berglund and A. Jayaraman, Hydrostatic-pressure dependence of the electronic properties of $\mathrm{VO}_{2}$ near the semiconductor-metal transition temperature, Phys. Rev. 185, 1034 (1969).

[44] J. P. Pouget, H. Launois, J. P. D’Haenens, P. Merenda, and T. M. Rice, Electron Localization Induced by Uniaxial Stress in Pure $\mathrm{VO}_{2}$, Phys. Rev. Lett. 35, 873 (1975).

[45] N. F. Quackenbush, H. Paik, M. J. Wahila, S. Sallis, M. E. Holtz, X. Huang, A. Ganose, B. J. Morgan, D. O. Scanlon, Y. Gu, F. Xue, L.-Q. Chen, G. E. Sterbinsky, C. Schlueter, T.-L. Lee, J. C. Woicik, J.-H. Guo, J. D. Brock, D. A. Muller, D. A. Arena, D. G. Schlom, and L. F. J. Piper, Stability of the M2 phase of vanadium dioxide induced by coherent epitaxial strain, Phys. Rev. B 94, 085105 (2016).

[46] M. Marezio, D. B. McWhan, J. P. Remeika, and P. D. Dernier, Structural aspects of the metal-insulator transitions in $\mathrm{Cr}$ doped $\mathrm{VO}_{2}$, Phys. Rev. B 5, 2541 (1972).

[47] T. M. Rice, H. Launois, and J. P. Pouget, Comment on " $\mathrm{VO}_{2}$ : Peierls or Mott-Hubbard? A View from Band Theory," Phys. Rev. Lett. 73, 3042 (1994).

[48] N. F. Mott and L. Friedman, Metal-insulator transitions in $\mathrm{VO}_{2}, \mathrm{Ti}_{2} \mathrm{O}_{3}$, and $\mathrm{Ti}_{2-x} \mathrm{~V}_{x} \mathrm{O}_{3}$, Philos. Mag. 30, 389 (1974).

[49] A. Pergament, Metal-insulator transition: The Mott criterion and coherence length, J. Phys.: Condens. Matter 15, 3217 (2003).

[50] Z. Zinamon and N. F. Mott, Metal-non-metal transitions in narrow band materials; crystal structure versus correlation, Philos. Mag. 21, 881 (1970).

[51] P. Werner, E. Gull, M. Troyer, and A. J. Millis, Spin Freezing Transition and Non-Fermi-Liquid Self-Energy in a ThreeOrbital Model, Phys. Rev. Lett. 101, 166405 (2008).

[52] M. M. Qazilbash, A. A. Schafgans, K. S. Burch, S. J. Yun, B. G. Chae, B. J. Kim, H. T. Kim, and D. N. Basov, Electrodynamics of the vanadium oxides $\mathrm{VO}_{2}$ and $\mathrm{V}_{2} \mathrm{O}_{3}$, Phys. Rev. B 77, 115121 (2008).

[53] S. Lee, K. Hippalgaonkar, F. Yang, J. Hong, C. Ko, J. Suh, K. Liu, K. Wang, J. J. Urban, X. Zhang, C. Dames, S. A. Hartnoll, O. Delaire, and J. Wu, Anomalously low electronic thermal conductivity in metallic vanadium dioxide, Science 355, 371 (2017).

[54] M. M. Qazilbash, M. Brehm, B.-G. Chae, P.-C. Ho, G. O. Andreev, B.-J. Kim, S. J. Yun, A. V. Balatsky, M. B. Maple, F. Keilmann, H.-T. Kim, and D. N. Basov, Mott transition in $\mathrm{VO}_{2}$ revealed by infrared spectroscopy and nano-imaging, Science 318, 1750 (2007).

[55] M. M. Qazilbash, K. S. Burch, D. Whisler, D. Shrekenhamer, B. G. Chae, H. T. Kim, and D. N. Basov, Correlated metallic state of vanadium dioxide, Phys. Rev. B 74, 205118 (2006).
[56] V. Eyert, The metal-insulator transitions of $\mathrm{VO}_{2}$ : $\mathrm{A}$ band theoretical approach, Ann. Phys. 11, 650 (2002).

[57] A. Liebsch, H. Ishida, and G. Bihlmayer, Coulomb correlations and orbital polarization in the metal-insulator transition of $\mathrm{VO}_{2}$, Phys. Rev. B 71, 085109 (2005).

[58] A. Continenza, S. Massidda, and M. Posternak, Self-energy corrections in $\mathrm{VO}_{2}$ within a model GW scheme, Phys. Rev. B 60, 15699 (1999).

[59] M. Gatti, F. Bruneval, V. Olevano, and L. Reining, Understanding Correlations in Vanadium Dioxide from First Principles, Phys. Rev. Lett. 99, 266402 (2007).

[60] R. Sakuma, T. Miyake, and F. Aryasetiawan, Quasiparticle band structure of vanadium dioxide, J. Phys.: Condens. Matter 21, 064226 (2009).

[61] M. A. Korotin, N. A. Skorikov, and V. I. Anisimov, Variation of orbital symmetry of the localized $3 d^{1}$ electron of the $\mathrm{V}^{4+}$ ion upon the metal-insulator transition in $\mathrm{VO}_{2}$, Phys. Met. Metallogr. 94, 17 (2002).

[62] A. V. Kozhevnikov, V. I. Anisimov, and M. A. Korotin, Calculation of the electronic structure of the vanadium dioxide $\mathrm{VO}_{2}$ in the monoclinic low-temperature phase M1 using the generalized transition state method, Phys. Met. Metallogr. 104, 215 (2007).

[63] X. Yuan, Y. Zhang, T. A. Abtew, P. Zhang, and W. Zhang, $\mathrm{VO}_{2}$ : Orbital competition, magnetism, and phase stability, Phys. Rev. B 86, 235103 (2012).

[64] R. Zhang, Q. S. Fu, C. Y. Yin, C. L. Li, X. H. Chen, G. Y. Qian, C. L. Lu, S. L. Yuan, X. J. Zhao, and H. Z. Tao, Understanding of metal-insulator transition in $\mathrm{VO}_{2}$ based on experimental and theoretical investigations of magnetic features, Sci. Rep. 8, 17093 (2018).

[65] R. Grau-Crespo, H. Wang, and U. Schwingenschlögl, Why the Heyd-Scuseria-Ernzerhof hybrid functional description of $\mathrm{VO}_{2}$ phases is not correct, Phys. Rev. B 86, 081101(R) (2012).

[66] J. E. Coulter, E. Manousakis, and A. Gali, Limitations of the hybrid functional approach to electronic structure of transition metal oxides, Phys. Rev. B 88, 041107(R) (2013).

[67] Z. Zhu and U. Schwingenschlögl, Comprehensive picture of $\mathrm{VO}_{2}$ from band theory, Phys. Rev. B 86, 075149 (2012).

[68] S. Xu, X. Shen, K. A. Hallman, R. F. Haglund, and S. T. Pantelides, Unified band-theoretic description of structural, electronic, and magnetic properties of vanadium dioxide phases, Phys. Rev. B 95, 125105 (2017).

[69] A. Moatti, R. Sachan, V. R. Cooper, and J. Narayan, Electrical transition in isostructural $\mathrm{VO}_{2}$ thin-film heterostructures, Sci. Rep. 9, 3009 (2019).

[70] G. Kotliar, S. Y. Savrasov, K. Haule, V. S. Oudovenko, O. Parcollet, and C. A. Marianetti, Electronic structure calculations with dynamical mean-field theory, Rev. Mod. Phys. 78, 865 (2006).

[71] A. S. Belozerov, M. A. Korotin, V. I. Anisimov, and A. I. Poteryaev, Monoclinic $M_{1}$ phase of $\mathrm{VO}_{2}$ : Mott-hubbard versus band insulator, Phys. Rev. B 85, 045109 (2012).

[72] C. Weber, D. D. O’Regan, N. D. M. Hine, M. C. Payne, G. Kotliar, and P. B. Littlewood, Vanadium Dioxide: A PeierlsMott Insulator Stable Against Disorder, Phys. Rev. Lett. 108, 256402 (2012).

[73] M. S. Laad, L. Craco, and E. Müller-Hartmann, Metalinsulator transition in rutile-based $\mathrm{VO}_{2}$, Phys. Rev. B 73, 195120 (2006). 
[74] W. H. Brito, M. C. O. Aguiar, K. Haule, and G. Kotliar, Dynamic electronic correlation effects in $\mathrm{NbO}_{2}$ as compared to $\mathrm{VO}_{2}$, Phys. Rev. B 96, 195102 (2017).

[75] N. Kumar, A. Rúa, F. E. Fernández, and S. Lysenko, Ultrafast diffraction conoscopy of the structural phase transition in $\mathrm{VO}_{2}$ : Evidence of two lattice distortions, Phys. Rev. B 95, 235157 (2017).

[76] X. Yuan, W. Zhang, and P. Zhang, Hole-lattice coupling and photoinduced insulator-metal transition in $\mathrm{VO}_{2}$, Phys. Rev. B 88, 035119 (2013).

[77] V. R. Morrison, R. P. Chatelain, K. L. Tiwari, A. Hendaoui, A. Bruhács, M. Chaker, and B. J. Siwick, A photoinduced metallike phase of monoclinic $\mathrm{VO}_{2}$ revealed by ultrafast electron diffraction, Science 346, 445 (2014).

[78] M. R. Otto, L. P. René de Cotret, D. A. Valverde-Chavez, K. L. Tiwari, N. Émond, M. Chaker, D. G. Cooke, and B. J. Siwick, How optical excitation controls the structure and properties of vanadium dioxide, Proc. Nat. Acad. Sci. USA 116, 450 (2019).

[79] Z. Tao, Tzong-Ru T. Han, S. D. Mahanti, P. M. Duxbury, F. Yuan, C.-Y. Ruan, K. Wang, and J. Wu, Decoupling of Structural and Electronic Phase Transitions in $\mathrm{VO}_{2}$, Phys. Rev. Lett. 109, 166406 (2012).

[80] S. A. Corr, D. P. Shoemaker, B. C. Melot, and R. Seshadri, Real-Space Investigation of Structural Changes at the MetalInsulator Transition in $\mathrm{VO}_{2}$, Phys. Rev. Lett. 105, 056404 (2010).

[81] J.-I. Umeda, S. Ashida, H. Kusumoto, and K. Narita, A new phase appearing in metal-semiconductor transition in $\mathrm{VO}_{2}$, J. Phys. Soc. Jpn. 21, 1461 (1966).

[82] J. Laverock, S. Kittiwatanakul, A. A. Zakharov, Y. R. Niu, B. Chen, S. A. Wolf, J. W. Lu, and K. E. Smith, Direct Observation of Decoupled Structural and Electronic Transitions and an Ambient Pressure Monocliniclike Metallic Phase of $\mathrm{VO}_{2}$, Phys. Rev. Lett. 113, 216402 (2014).

[83] T. Yao, X. Zhang, Z. Sun, S. Liu, Y. Huang, Yi Xie, C. Wu, X. Yuan, W. Zhang, Z. Wu, G. Pan, F. Hu, L. Wu, Q. Liu, and $\mathrm{S}$. Wei, Understanding the Nature of the Kinetic Process in a $\mathrm{VO}_{2}$ Metal-Insulator Transition, Phys. Rev. Lett. 105, 226405 (2010).

[84] D. Lee, B. Chung, Y. Shi, G.-Y. Kim, N. Campbell, F. Xue, K. Song, S.-Y. Choi, J. P. Podkaminer, T. H. Kim, P. J. Ryan, J.-W. Kim, T. R. Paudel, J.-H. Kang, J. W. Spinuzzi, D. A. Tenne, E. Y. Tsymbal, M. S. Rzchowski, L. Q. Chen, J. Lee, and C. B. Eom, Isostructural metal-insulator transition in $\mathrm{VO}_{2}$, Science 362, 1037 (2018).

[85] E. Arcangeletti, L. Baldassarre, D. Di Castro, S. Lupi, L. Malavasi, C. Marini, A. Perucchi, and P. Postorino, Evidence of a Pressure-Induced Metallization Process in Monoclinic $\mathrm{VO}_{2}$, Phys. Rev. Lett. 98, 196406 (2007).

[86] A. X. Gray, J. Jeong, N. P. Aetukuri, P. Granitzka, Z. Chen, R. Kukreja, D. Higley, T. Chase, A. H. Reid, H. Ohldag, M. A. Marcus, A. Scholl, A. T. Young, A. Doran, C. A. Jenkins, P. Shafer, E. Arenholz, M. G. Samant, S. S. P. Parkin, and H. A. Dürr, Correlation-Driven Insulator-Metal Transition in NearIdeal Vanadium Dioxide Films, Phys. Rev. Lett. 116, 116403 (2016).

[87] N. B. Aetukuri, A. X. Gray, M. Drouard, M. Cossale, L. Gao, A. H. Reid, R. Kukreja, H. Ohldag, C. A. Jenkins, E. Arenholz, K. P. Roche, H. A. Dürr, M. G. Samant, and S. S. P. Parkin,
Control of the metal-insulator transition in vanadium dioxide by modifying orbital occupancy, Nat. Phys. 9, 661 (2013).

[88] T. J. Huffman, C. Hendriks, E. J. Walter, J. Yoon, H. Ju, R. Smith, G. L. Carr, H. Krakauer, and M. M. Qazilbash, Insulating phases of vanadium dioxide are Mott-Hubbard insulators, Phys. Rev. B 95, 075125 (2017).

[89] J. D. Budai, J. Hong, M. E. Manley, E. D. Specht, C. W. Li, J. Z. Tischler, D. L. Abernathy, A. H. Said, B. M. Leu, L. A. Boatner, R. J. McQueeney, and O. Delaire, Metallization of vanadium dioxide driven by large phonon entropy, Nature (London) 515, 535 (2014).

[90] W. Paul, The present position of theory and experiment for $\mathrm{VO}_{2}$, Mater. Res. Bull. 5, 691 (1970).

[91] P. Lederer, H. Launois, J. P. Pouget, A. Casalot, and G. Villeneuve, Contribution to the study of the metal-insulator transition in the $\mathrm{V}_{1-x} \mathrm{Nb}_{x} \mathrm{O}_{2}$ system. III: Theoretical discussion, J. Phys. Chem. Solids 33, 1969 (1972).

[92] M. Gatti, G. Panaccione, and L. Reining, Effects of LowEnergy Excitations on Spectral Properties at Higher Binding Energy: The Metal-Insulator Transition of $\mathrm{VO}_{2}$, Phys. Rev. Lett. 114, 116402 (2015).

[93] I.-H. Hwang, Z. Jin, C.-I. Park, and S.-W. Han, The influence of structural disorder and phonon on metal-to-insulator transition of $\mathrm{VO}_{2}$, Sci. Rep. 7, 14802 (2017).

[94] D. B. McWhan, J. P. Remeika, J. P. Maita, H. Okinaka, K. Kosuge, and S. Kachi, Heat capacity of vanadium oxides at low temperature, Phys. Rev. B 7, 326 (1973).

[95] D. B. McWhan, M. Marezio, J. P. Remeika, and P. D. Dernier, X-ray diffraction study of metallic $\mathrm{VO}_{2}$, Phys. Rev. B 10, 490 (1974).

[96] R. Srivastava and L. L. Chase, Raman Spectrum of Semiconducting and Metallic $\mathrm{VO}_{2}$, Phys. Rev. Lett. 27, 727 (1971).

[97] C. Sommers, R. De Groot, D. Kaplan, and A. Zylbersztejn, Cluster calculations of the electronic $d$-states in $\mathrm{VO}_{2}$, J. Physique Lett. 36, 157 (1975).

[98] G. J. Hyland, On the electronic phase transitions in the lower oxides of vanadium, J. Phys. C 1, 189 (1968)

[99] M. Gupta, A. J. Freeman, and D. E. Ellis, Electronic structure and lattice instability of metallic $\mathrm{VO}_{2}$, Phys. Rev. B 16, 3338 (1977).

[100] G. Stefanovich, A. Pergament, and D. Stefanovich, Electrical switching and Mott transition in $\mathrm{VO}_{2}$, J. Phys.: Condens. Matter 12, 8837 (2000).

[101] D. Maurer, A. Leue, R. Heichele, and V. Müller, Elastic behavior near the metal-insulator transition of $\mathrm{VO}_{2}$, Phys. Rev. B 60, 13249 (1999).

[102] F. Pintchovski, W.S. Glaunsinger, and A. Navrotsky, Experimental study of the electronic and lattice contributions to the $\mathrm{VO}_{2}$ transition, J. Phys. Chem. Solids 39, 941 (1978).

[103] S. Wall, D. Wegkamp, L. Foglia, K. Appavoo, J. Nag, R. F. Haglund Jr., J. Stahler, and M. Wolf, Ultrafast changes in lattice symmetry probed by coherent phonons, Nat. Commun. 3, 721 (2012).

[104] C. Kübler, H. Ehrke, R. Huber, R. Lopez, A. Halabica, R. F. Haglund, and A. Leitenstorfer, Coherent Structural Dynamics and Electronic Correlations During an Ultrafast Insulator-toMetal Phase Transition in $\mathrm{VO}_{2}$, Phys. Rev. Lett. 99, 116401 (2007). 
[105] D. Wegkamp and J. Stähler, Ultrafast dynamics during the photoinduced phase transition in $\mathrm{VO}_{2}$, Prog. Surf. Sci. 90, 464 (2015).

[106] P. Schilbe and D. Maurer, Lattice dynamics in $\mathrm{VO}_{2}$ near the metal-insulator transition, Mater. Sci. Eng.: A 370, 449 (2004).

[107] J. R. Brews, Symmetry considerations and the vanadium dioxide phase transition, Phys. Rev. B 1, 2557 (1970).

[108] A. Cavalleri, C. Tóth, C. W. Siders, J. A. Squier, F. Ráksi, P. Forget, and J. C. Kieffer, Femtosecond Structural Dynamics in $\mathrm{VO}_{2}$ During an Ultrafast Solid-Solid Phase Transition, Phys. Rev. Lett. 87, 237401 (2001).

[109] Z. He and A. J. Millis, Photoinduced phase transitions in narrow-gap Mott insulators: The case of $\mathrm{VO}_{2}$, Phys. Rev. B 93, 115126 (2016).

[110] Y. H. Matsuda, D. Nakamura, A. Ikeda, S. Takeyama, Y. Muraoka, and Y. Suga, Magnetic-field-induced insulator-metal transition in W-doped $\mathrm{VO}_{2}$ at $500 \mathrm{~T}$, arXiv:2001.08580.

[111] H. S. Choe, J. Suh, C. Ko, K. Dong, S. Lee, J. Park, Y. Lee, K. Wang, and J. Wu, Enhancing modulation of thermal conduction in vanadium dioxide thin film by nanostructured nanogaps, Sci. Rep. 7, 7131 (2017).

[112] J. B. Goodenough, Direct cation-cation interactions in several oxides, Phys. Rev. 117, 1442 (1960).

[113] A. S. Barker, H. W. Verleur, and H. J. Guggenheim, Infrared Optical Properties of Vanadium Dioxide Above and Below the Transition Temperature, Phys. Rev. Lett. 17, 1286 (1966).

[114] D. Adler, Insulating and Metallic States in Transition Metal Oxides (Academic Press, San Diego, 1968), pp. 1-113.

[115] H. Sasaki and A. Watanabe, A new growing method for $\mathrm{VO}_{2}$ single crystals, J. Phys. Soc. Jpn. 19, 1748 (1964).

[116] L. A. Ladd and W. Paul, Optical and transport properties of high quality crystals of $\mathrm{V}_{2} \mathrm{O}_{4}$ near the metallic transition temperature, Solid State Commun. 7, 425 (1969).

[117] C. R. Everhart and J. B. MacChesney, Anisotropy in the electrical resistivity of vanadium dioxide single crystals, J. Appl. Phys. 39, 2872 (1968).

[118] P. F. Bongers, Anisotropy of the electrical conductivity of $\mathrm{VO}_{2}$ single crystals, Solid State Commun. 3, 275 (1965).

[119] W.-T. Liu, J. Cao, W. Fan, Z. Hao, M. C. Martin, Y. R. Shen, J. Wu, and F. Wang, Intrinsic optical properties of vanadium dioxide near the insulator-metal transition, Nano Lett. 11, 466 (2011).

[120] M. Nakano, K. Shibuya, D. Okuyama, T. Hatano, S. Ono, M. Kawasaki, Y. Iwasa, and Y. Tokura, Collective bulk carrier delocalization driven by electrostatic surface charge accumulation, Nature (London) 487, 459 (2012).

[121] D. Okuyama, M. Nakano, S. Takeshita, H. Ohsumi, S. Tardif, K. Shibuya, T. Hatano, H. Yumoto, T. Koyama, H. Ohashi, M. Takata, M. Kawasaki, T. Arima, Y. Tokura, and Y. Iwasa, Gate-tunable gigantic lattice deformation in $\mathrm{VO}_{2}$, Appl. Phys. Lett. 104, 023507 (2014).

[122] G. J. Hyland and A. W. B. Taylor, Clausius-Clapeyron equation and the $\mathrm{V}_{2} \mathrm{O}_{4}, \mathrm{~V}_{2} \mathrm{O}_{3}$ phase transitions, J. Phys. Soc. Jpn. 21, 819B (1966).

[123] T. Kawakubo and T. Nakagawa, Phase transition in $\mathrm{VO}_{2}$, J. Phys. Soc. Jpn. 19, 517 (1964)

[124] G. V. Chandrashekhar, H. L. C. Barros, and J. M. Honig, Heat capacity of $\mathrm{VO}_{2}$ single crystals, Mater. Res. Bull. 8, 369 (1973).
[125] T. Mitsuishi, On the phase transformation of $\mathrm{VO}_{2}$, Jpn. J. Appl. Phys. 6, 1060 (1967).

[126] B.-J. Kim, Y. W. Lee, S. Choi, J.-W. Lim, S. J. Yun, H.-T. Kim, T.-J. Shin, and H.-S. Yun, Micrometer X-ray diffraction study of $\mathrm{VO}_{2}$ films: Separation between metal-insulator transition and structural phase transition, Phys. Rev. B 77, 235401 (2008).

[127] J. Nag, R. F. Haglund, E. Andrew Payzant, and K. L. More, Non-congruence of thermally driven structural and electronic transitions in $\mathrm{VO}_{2}$, J. Appl. Phys. 112, 103532 (2012).

[128] S. Zhang, J. Y. Chou, and L. J. Lauhon, Direct correlation of structural domain formation with the metal insulator transition in a $\mathrm{VO}_{2}$ nanobeam, Nano Lett. 9, 4527 (2009).

[129] H.-T. Kim, Y. W. Lee, B.-J. Kim, B.-G. Chae, S. J. Yun, K.-Y. Kang, K.-J. Han, K.-J. Yee, and Y.-S. Lim, Monoclinic and Correlated Metal Phase in $\mathrm{VO}_{2}$ as Evidence of the Mott Transition: Coherent Phonon Analysis, Phys. Rev. Lett. 97, 266401 (2006).

[130] T. L. Cocker, L. V. Titova, S. Fourmaux, G. Holloway, H.-C. Bandulet, D. Brassard, J.-C. Kieffer, M. A. El Khakani, and F. A. Hegmann, Phase diagram of the ultrafast photoinduced insulator-metal transition in vanadium dioxide, Phys. Rev. B 85, 155120 (2012).

[131] S. Kumar, J. P. Strachan, M. D. Pickett, A. Bratkovsky, Y. Nishi, and R. S. Williams, Sequential electronic and structural transitions in $\mathrm{VO}_{2}$ observed using x-ray absorption spectromicroscopy, Adv. Mater. 26, 7505 (2014).

[132] A. V. Ilinskiy, O. E. Kvashenkina, and E. B. Shadrin, Nature of the electronic component of the thermal phase transition in $\mathrm{VO}_{2}$ films, Semiconductors 46, 1171 (2012).

[133] A. V. Ilinskiy, O. E. Kvashenkina, and E. B. Shadrin, Phase transition and correlation effects in vanadium dioxide, Semiconductors 46, 422 (2012).

[134] C. Chen, R. Wang, L. Shang, and C. Guo, Gate-field-induced phase transitions in $\mathrm{VO}_{2}$ : Monoclinic metal phase separation and switchable infrared reflections, Appl. Phys. Lett. 93, 171101 (2008).

[135] D. C. Mattis and W. D. Langer, Role of Phonons and Band Structure in Metal-Insulator Phase Transition, Phys. Rev. Lett. 25, 376 (1970).

[136] C. J. Hearn, Phonon softening and the metal-insulator transition in $\mathrm{VO}_{2}$, J. Phys. C 5, 1317 (1972).

[137] J. M. Tomczak, F. Aryasetiawan, and S. Biermann, Effective bandstructure in the insulating phase versus strong dynamical correlations in metallic $\mathrm{VO}_{2}$, Phys. Rev. B 78, 115103 (2008).

[138] T. Kawakubo, Crystal distortion and electric and magnetic transition in $\mathrm{VO}_{2}$, J. Phys. Soc. Jpn. 20, 516 (1965).

[139] C.J. Hearn, The metal-insulator transition in $\mathrm{VO}_{2}$, Phys. Lett. A 38, 447 (1972).

[140] G. J. Hyland, Semiconductor $\rightleftarrows$ metal phase transitions, J. Solid State Chem. 2, 318 (1970).

[141] D. Adler, J. Feinleib, H. Brooks, and W. Paul, Semiconductorto-metal transitions in transition-metal compounds, Phys. Rev. 155, 851 (1967).

[142] T. K. Mitra, S. Chatterjee, and G. J. Hyland, A L.C.O.A.O approach to the band structure of rutile $\mathrm{VO}_{2}$, Phys. Lett. A 37, 221 (1971).

[143] S. Chattejee, T. K. Mitra, and G. J. Hyland, A.P.W. band structure of metallic $\mathrm{VO}_{2}$, Phys. Lett. A 42, 56 (1972). 
[144] E. Caruthers, L. Kleinman, and H. I. Zhang, Energy bands of metallic $\mathrm{VO}_{2}$, Phys. Rev. B 7, 3753 (1973).

[145] E. Caruthers and L. Kleinman, Energy bands of semiconducting $\mathrm{VO}_{2}$, Phys. Rev. B 7, 3760 (1973).

[146] S. M. Woodley, The mechanism of the displacive phase transition in vanadium dioxide, Chem. Phys. Lett. 453, 167 (2008).

[147] M. Netsianda, P. E. Ngoepe, C. R. A. Catlow, and S. M. Woodley, The displacive phase transition of vanadium dioxide and the effect of doping with tungsten, Chem. Mater. 20, 1764 (2008).

[148] A. Tselev, I. A. Luk'yanchuk, I. N. Ivanov, J. D. Budai, J. Z. Tischler, E. Strelcov, A. Kolmakov, and S. V. Kalinin, Symmetry relationship and strain-induced transitions between insulating M1 and M2 and metallic R phases of vanadium dioxide, Nano Lett. 10, 4409 (2010).

[149] A. M. de Graaf and R. Luzzi, Crystallographic distortion, electron-electron interaction, and the metal-nonmetal transition, Helv. Phys. Acta 41, 764 (1968).

[150] D. Paquet and P. Leroux-Hugon, Electron correlations and electron-lattice interactions in the metal-insulator, ferroelastic transition in $\mathrm{VO}_{2}$ : A thermodynamical study, Phys. Rev. B 22, 5284 (1980).

[151] J. Shi, R. Bruinsma, and A. R. Bishop, Theory of vanadium dioxide, Synth. Met. 43, 3527 (1991).

[152] M. Sandri, M. Capone, and M. Fabrizio, Finite-temperature Gutzwiller approximation and the phase diagram of a toy model for $\mathrm{V}_{2} \mathrm{O}_{3}$, Phys. Rev. B 87, 205108 (2013).

[153] A. O. Sboychakov, A. L. Rakhmanov, and K. I. Kugel, Effect of electron-lattice interaction on the phase separation in strongly correlated electron systems with two types of charge carriers, J. Phys.: Condens. Matter 22, 415601 (2010).

[154] M. W. Haverkort, Z. Hu, A. Tanaka, W. Reichelt, S. V. Streltsov, M. A. Korotin, V. I. Anisimov, H. H. Hsieh, H.-J. Lin, C. T. Chen, D. I. Khomskii, and L. H. Tjeng, OrbitalAssisted Metal-Insulator Transition in $\mathrm{VO}_{2}$, Phys. Rev. Lett. 95, 196404 (2005).

[155] B.-C. Shih, T. A. Abtew, X. Yuan, W. Zhang, and P. Zhang, Screened Coulomb interactions of localized electrons in transition metals and transition-metal oxides, Phys. Rev. B 86, 165124 (2012).

[156] A. Fujimori, I. Hase, H. Namatame, Y. Fujishima, Y. Tokura, H. Eisaki, S. Uchida, K. Takegahara, and F. M. F. de Groot, Evolution of the Spectral Function in Mott-Hubbard Systems with $d^{1}$ Configuration, Phys. Rev. Lett. 69, 1796 (1992).

[157] L. de' Medici, J. Mravlje, and A. Georges, Janus-Faced Influence of Hund's Rule Coupling in Strongly Correlated Materials, Phys. Rev. Lett. 107, 256401 (2011).

[158] A. P. Levanyuk and D. G. Sannikov, Improper ferroelectrics, Sov. Phys. Uspekhi 17, 199 (1974).

[159] G. J. Hyland, Lattice polarization and coulomb energies in VO $_{2}$, Philos. Mag. 20, 837 (1969).

[160] B. Lazarovits, K. Kim, K. Haule, and G. Kotliar, Effects of strain on the electronic structure of $\mathrm{VO}_{2}$, Phys. Rev. B 81, 115117 (2010).

[161] C. Sommers and S. Doniach, First principles calculation of the intra-atomic correlation energy in $\mathrm{VO}_{2}$, Solid State Commun. 28, 133 (1978).

[162] K. Okazaki, H. Wadati, A. Fujimori, M. Onoda, Y. Muraoka, and Z. Hiroi, Photoemission study of the metal-insulator transition in $\mathrm{VO}_{2} / \mathrm{TiO}_{2}(001)$ : Evidence for strong electron- electron and electron-phonon interaction, Phys. Rev. B 69, 165104 (2004).

[163] M. van Veenendaal, Ultrafast photoinduced insulator-to-metal transitions in vanadium dioxide, Phys. Rev. B 87, 235118 (2013).

[164] S. Lysenko, N. Kumar, A. Rúa, J. Figueroa, J. Lu, and F. Fernández, Ultrafast structural dynamics of $\mathrm{VO}_{2}$, Phys. Rev. B 96, 075128 (2017).

[165] A. Georges, G. Kotliar, W. Krauth, and M. J. Rozenberg, Dynamical mean-field theory of strongly correlated fermion systems and the limit of infinite dimensions, Rev. Mod. Phys. 68, 13 (1996).

[166] M. Caffarel and W. Krauth, Exact Diagonalization Approach to Correlated Fermions in Infinite Dimensions: Mott Transition and Superconductivity, Phys. Rev. Lett. 72, 1545 (1994).

[167] C. Weber, A. Amaricci, M. Capone, and P. B. Littlewood, Augmented hybrid exact-diagonalization solver for dynamical mean field theory, Phys. Rev. B 86, 115136 (2012).

[168] M. Capone, L. de' Medici, and A. Georges, Solving the dynamical mean-field theory at very low temperatures using the Lanczos exact diagonalization, Phys. Rev. B 76, 245116 (2007).

[169] I. A. Mogunov, F. Fernández, S. Lysenko, A. J. Kent, A. V. Scherbakov, A. M. Kalashnikova, and A. V. Akimov, Ultrafast Insulator-Metal Transition in $\mathrm{VO}_{2}$ Nanostructures Assisted by Picosecond Strain Pulses, Phys. Rev. Appl. 11, 014054 (2019).

[170] J. Cao, E. Ertekin, V. Srinivasan, W. Fan, S. Huang, H. Zheng, J. W. L. Yim, D. R. Khanal, D. F. Ogletree, J. C. Grossman, and $\mathrm{J}$. Wu, Strain engineering and one-dimensional organization of metal-insulator domains in single-crystal vanadium dioxide beams, Nat. Nanotechnol. 4, 732 (2009).

[171] B.A. Strukov and A. P. Levanyuk, Ferroelectric Phenomena In Crystals: Physical Foundations (Springer, Berlin, 1998).

[172] M. Sandri and M. Fabrizio, Nonequilibrium gap collapse near a first-order Mott transition, Phys. Rev. B 91, 115102 (2015).

[173] F. Grandi, A. Amaricci, M. Capone, and M. Fabrizio, Correlation-driven Lifshitz transition and orbital order in a two-band Hubbard model, Phys. Rev. B 98, 045105 (2018).

[174] T. C. Koethe, Z. Hu, M. W. Haverkort, C. SchüßlerLangeheine, F. Venturini, N. B. Brookes, O. Tjernberg, W. Reichelt, H. H. Hsieh, H.-J. Lin, C. T. Chen, and L. H. Tjeng, Transfer of Spectral Weight and Symmetry Across the Metal-Insulator Transition in $\mathrm{VO}_{2}$, Phys. Rev. Lett. 97, 116402 (2006).

[175] A. S. Belozerov, A. I. Poteryaev, and V. I. Anisimov, Evidence for strong Coulomb correlations in the metallic phase of vanadium dioxide, JETP Lett. 93, 70 (2011).

[176] L. A. L. de Almeida, G. S. Deep, A. M. N. Lima, and H. Neff, Thermal dynamics of $\mathrm{VO}_{2}$ films within the metal-insulator transition: Evidence for chaos near percolation threshold, Appl. Phys. Lett. 77, 4365 (2000).

[177] R. Lopez, T. E. Haynes, L. A. Boatner, L. C. Feldman, and R. F. Haglund, Size effects in the structural phase transition of $\mathrm{VO}_{2}$ nanoparticles, Phys. Rev. B 65, 224113 (2002).

[178] M. Pan, J. Liu, H. Zhong, S. Wang, Z. F. Li, X. Chen, and W. Lu, Raman study of the phase transition in $\mathrm{VO}_{2}$ thin films, J. Cryst. Growth 268, 178 (2004).

[179] J. H. Claassen, J. W. Lu, K. G. West, and S. A. Wolf, Relaxation dynamics of the metal-semiconductor transition in $\mathrm{VO}_{2}$ thin films, Appl. Phys. Lett. 96, 132102 (2010). 
[180] B. Viswanath and S. Ramanathan, Direct in situ observation of structural transition driven actuation in $\mathrm{VO}_{2}$ utilizing electron transparent cantilevers, Nanoscale 5, 7484 (2013).

[181] T. Schneider and E. Stoll, Molecular-Dynamics Study of a Two-Dimensional Ferrodistortive XY Model with Quartic Anisotropy, Phys. Rev. Lett. 36, 1501 (1976).

[182] J. V. José, L. P. Kadanoff, S. Kirkpatrick, and D. R. Nelson, Renormalization, vortices, and symmetry-breaking perturbations in the two-dimensional planar model, Phys. Rev. B 16, 1217 (1977).

[183] J. Lou, A. W. Sandvik, and L. Balents, Emergence of U(1) Symmetry in the $3 \mathrm{~d} x y$ Model with $Z_{q}$ Anisotropy, Phys. Rev. Lett. 99, 207203 (2007).

[184] K. Binder and D. Heermann, Monte Carlo Simulation in Statistical Physics, 6th ed., Graduate Text in Physics (Springer, Berlin, 2019).

[185] L. Chen, Z. Xiang, C. Tinsman, T. Asaba, Q. Huang, H. Zhou, and $\mathrm{L}$. Li, Enhancement of thermal conductivity across the metal-insulator transition in vanadium dioxide, Appl. Phys. Lett. 113, 061902 (2018).

[186] T. A. Mellan, H. Wang, U. Schwingenschlögl, and R. GrauCrespo, Origin of the transition entropy in vanadium dioxide, Phys. Rev. B 99, 064113 (2019).

[187] R. F. Janninck and D. H. Whitmore, Electrical conductivity and thermoelectric power of niobium dioxide, J. Phys. Chem. Solids 27, 1183 (1966).

[188] M. R. Beebe, J. M. Klopf, Y. Wang, S. Kittiwatanakul, J. Lu, S. A. Wolf, and R. A. Lukaszew, Time-resolved light-induced insulator-metal transition in niobium dioxide and vanadium dioxide thin films, Opt. Mater. Express 7, 213 (2017).

[189] K. Seta and K. Naito, Calorimetric study of the phase transition in $\mathrm{NbO}_{2}$, J. Chem. Thermodyn. 14, 921 (1982).

[190] C. N. R. Rao, G. R. Rao, and G. V. S. Rao, Semiconductormetal transitions in $\mathrm{NbO}_{2}$ and $\mathrm{Nb}_{1-x} \mathrm{~V}_{x} \mathrm{O}_{2}$, J. Solid State Chem. 6, 340 (1973).

[191] J. B. Goodenough, Metallic oxides, Prog. Solid State Chem. 5, 145 (1971)

[192] P. Tolédano and J.-C. Tolédano, Order-parameter symmetries for the phase transitions of nonmagnetic secondary and higherorder ferroics, Phys. Rev. B 16, 386 (1977).
[193] T. Sakata, K. Sakata, and I. Nishida, Study of phase transition in $\mathrm{NbO}_{2}$, Phys. Stat. Solidi (B) 20, K155 (1967).

[194] A. R. Dhamdhere, T. Hadamek, A. B. Posadas, A. A. Demkov, and D. J. Smith, Structural characterization of niobium oxide thin films grown on $\mathrm{SrTiO}_{3}(111)$ and $(\mathrm{La}, \mathrm{Sr})(\mathrm{Al}, \mathrm{Ta}) \mathrm{O}_{3}(111)$ substrates, J. Appl. Phys. 120, 245302 (2016).

[195] A. A. Bolzan, C. Fong, B. J. Kennedy, and C. J. Howard, A powder neutron diffraction study of semiconducting and metallic niobium dioxide, J. Solid State Chem. 113, 9 (1994).

[196] Z. Hiroi, Structural instability of the rutile compounds and its relevance to the metal-insulator transition of $\mathrm{VO}_{2}$, Prog. Solid State Chem. 43, 47 (2015)

[197] K. Sakata, Note on the phase transition in $\mathrm{NbO}_{2}$, J. Phys. Soc. Jpn. 26, 582 (1969).

[198] G. Bélanger, J. Destry, G. Perluzzo, and P. M. Raccah, Electron transport in single crystals of niobium dioxide, Can. J. Phys. 52, 2272 (1974).

[199] M. J. Wahila, G. Paez, C. N. Singh, A. Regoutz, S. Sallis, M. J. Zuba, J. Rana, M. B. Tellekamp, J. E. Boschker, T. Markurt, J. E. N. Swallow, L. A. H. Jones, T. D. Veal, W. Yang, T.-L. Lee, F. Rodolakis, J. T. Sadowski, D. Prendergast, W.-C. Lee, W. A. Doolittle, and L. F. J. Piper, Evidence of a second-order Peierls-driven metal-insulator transition in crystalline $\mathrm{NbO}_{2}$, Phys. Rev. Mat. 3, 074602 (2019).

[200] J. Haines, J. M. Léger, A. S. Pereira, D. Häusermann, and M. Hanfland, High-pressure structural phase transitions in semiconducting niobium dioxide, Phys. Rev. B 59, 13650 (1999).

[201] V. Eyert, The metal-insulator transition of $\mathrm{NbO}_{2}$ : An embedded Peierls instability, EPL 58, 851 (2002).

[202] A. O'Hara, T. N. Nunley, A. B. Posadas, S. Zollner, and A. A. Demkov, Electronic and optical properties of $\mathrm{NbO}_{2}$, J. Appl. Phys. 116, 213705 (2014).

[203] A. O'Hara and A. A. Demkov, Nature of the metalinsulator transition in $\mathrm{NbO}_{2}$, Phys. Rev. B 91, 094305 (2015).

[204] F. J. Wong, N. Hong, and S. Ramanathan, Orbital splitting and optical conductivity of the insulating state of $\mathrm{NbO}_{2}$, Phys. Rev. B 90, 115135 (2014). 